\title{
THE TOPOLOGICAL ASYMPTOTIC EXPANSION FOR THE QUASI-STOKES PROBLEM
}

\author{
MaAtoug Hassine $^{1}$ And Mohamed Masmoudi ${ }^{2}$
}

\begin{abstract}
In this paper, we propose a topological sensitivity analysis for the Quasi-Stokes equations. It consists in an asymptotic expansion of a cost function with respect to the creation of a small hole in the domain. The leading term of this expansion is related to the principal part of the operator. The theoretical part of this work is discussed in both two and three dimensional cases. In the numerical part, we use this approach to optimize the locations of a fixed number of air injectors in an eutrophized lake.
\end{abstract}

Mathematics Subject Classification. 49Q10, 49Q12, 74P05, 74P10, 74P15.

Received August 28, 2002. Revised November 6, 2003.

\section{INTRODUCTION}

The goal of topological optimization is to find an optimal design even with a priori poor information on the optimal shape. Unlike the case of classical optimization, the topology of the structure may change during the optimization process, as for example by the inclusion of holes.

Most of the known results in this field concern structural mechanics. In such cases, classical topology optimization involves relaxed formulations or homogenization (see e.g. $[2,3,5,9,26,30]$ ). This method leads to a Neumann condition on the unknown boundary. This boundary condition is quite natural in structural mechanics but this is not the case in fluid dynamics. In that direction, global optimization techniques like genetic algorithms or simulated annealing, have been proposed (see e.g. [36]). But these methods are very slow and can hardly be applied to industrial problems.

The recently introduced notion of topological sensitivity gives new perspectives on shape optimization. It provides an asymptotic expansion of a cost function with respect to the creation of a small hole in the domain. To present the basic idea, we consider $\Omega$ a domain of $\mathbb{R}^{N}, N=2,3$ and $j(\Omega)=J\left(\Omega, u_{\Omega}\right)$ a cost function to be minimized, where $u_{\Omega}$ is the solution to a given PDE problem defined in $\Omega$. For $\varepsilon>0$, let $\Omega_{\varepsilon}=\Omega \backslash \overline{\left(x_{0}+\varepsilon \omega\right)}$ be the domain obtained by removing a small part $\overline{\left(x_{0}+\varepsilon \omega\right)}$ from $\Omega$, where $x_{0} \in \Omega$ and $\omega \subset \mathbb{R}^{N}$ is a fixed bounded domain containing the origin. Then, an asymptotic expansion of the function $j$ is obtained in the following

Keywords and phrases. Topological optimization, topological sensitivity, Quasi-Stokes equations, topological gradient, shape optimization.

1 ENIT-LAMSIN \& FSM, Campus Universitaire, Le Belvédaire BP 37, 1002 Tunis, Tunisia;

e-mail: maatoug.hassine@enit.rnu.tn

2 Mathématiques pour l'Industrie et la Physique, UMR 5640, Université de Paul Sabatier, 118 route de Narbonne, 31032 Toulouse Cedex 4, France. 
form:

$$
\begin{aligned}
& j\left(\Omega_{\varepsilon}\right)=j(\Omega)+\rho(\varepsilon) \delta j\left(x_{0}\right)+o(\rho(\varepsilon)) \\
& \lim _{\varepsilon \rightarrow 0} \rho(\varepsilon)=0, \quad \rho(\varepsilon)>0
\end{aligned}
$$

where $\rho(\varepsilon)$ is a scalar function known explicitly and $\delta j\left(x_{0}\right)$ is easy to compute at each point $x_{0}$ of $\Omega$.

The topological sensitivity $\delta j\left(x_{0}\right)$ gives us the information where to create holes, in fact if $\delta j\left(x_{0}\right)<0$, then $j\left(\Omega_{\varepsilon}\right)<j(\Omega)$ for small $\varepsilon$. The function $\delta j$ can be used as a descent direction of the domain optimization process.

The optimality condition $\delta j\left(x_{0}\right) \geq 0$ in the domain recalls the one obtained by Buttazzo-Dal Maso [5] for the Laplace equation using the homogenization theory. The notion of topological asymptotic gives an interesting alternative to homogenization methods and genetic algorithms: its applications field is very large and using topological sensitivity information, one can build fast algorithms.

The total energy variation with respect to the creation of a small hole is well known [16]. Schumacher introduced the bubble method that uses this energy variation for topological optimization in [35]. Then Sokolowski extended this idea to more general cost functions using the adjoint approach in [37] but still with Neumann boundary condition. A topological sensitivity framework using an adaptation of the adjoint method [7,29] and a truncation technique was introduced in [29] in the case of the Laplace equation with a circular hole and a Dirichlet condition on the boundary of the hole. It was generalized in [15] to the elasticity equations in the case of arbitrary shaped holes. Recently, the same technique is adapted, using a Dirichlet boundary condition and non circular holes, to the Poisson equation in [23] and to Stokes equations in [24].

All these contributions concern operators which symbol is an homogeneous polynomial. The goal of this paper is to address the situations where non homogeneous polynomials arise. We will illustrate our approach by the Quasi-Stokes equations case. The basic idea is to say that the leading term of the topological expansion is given by the elementary solution of the principal part of the operator. The theoretical part of this work is discussed in both two and three dimensional cases. Such an expansion is obtained for a large class of cost functions and arbitrary shaped holes.

In the numerical part, we consider the aeration process of eutrophized lakes. Eutrophication leads to a 3layer situation, the bottom layer being quite poor in oxygen necessary to aquatic life [1]. The aeration process consists in inserting air by the means of injectors located at the bottom of the lake in order to generate a vertical motion mixing up the water of the bottom with that in the top, thus oxygenating the lower part by bringing it in contact with the surface air. A simplified model based on incompressible Quasi-Stokes equations is used, only considering the liquid phase, which is the dominant one. The injected air is taken into account through local boundary conditions for the velocity on the injectors holes. We aim to optimize the injectors location in order to generate the best motion in the fluid with respect to the aeration purpose. The main idea is to compute the asymptotic topological expansion with respect to the insertion of an injector. The injector is modeled as a small hole $\omega_{\varepsilon}$ around a point $x_{0}$, having an injection velocity $\mathcal{U}_{\mathrm{inj}}$. The best locations and orientations are the one for which the cost function decrease most, i.e. the sensitivity is as negative as possible. Numerical tests clearly indicate the approach to be quite efficient.

An outline of the paper is as follows. In Section 2, we recall briefly the adaptation of adjoint method to the topological optimization. In Section 3, we derive the Quasi-Stokes equations and we give a description of the shape optimization problem that we consider. Next in Section 4 the truncation technique is applied to the problem. The main results are presented in Section 5. An asymptotic expansion is given in a general form, for a large class of cost functions and arbitrary shaped holes. In Section 6, we present, for the two dimensional case, some numerical experiments validating the above analysis. Finally in Section 7, some background materials related to the Stokes and Quasi-Stokes equations are reviewed.

\section{THE GENERALIZED ADJOINT METHOD}

In this section, we recall the fundamental results introduced in $[14,29]$ which extends the adjoint method [7] to the topology shape optimization. 
Let $\mathcal{V}$ be a fixed Hilbert space. For $\varepsilon \geq 0$, let $a_{\varepsilon}$ be a bilinear, symmetric, continuous and coercive form on $\mathcal{V}$ and $l_{\varepsilon}$ be a linear and continuous form on $\mathcal{V}$, that is, there exist constants $M>0, \gamma>0$ and $L>0$, independent of $\varepsilon$ such that for all $\varepsilon \geq 0$,

$$
\begin{array}{ll}
a_{\varepsilon}(u, v) \leq M\|u\|\|v\|, & \forall u, v \in \mathcal{V} \\
a_{\varepsilon}(u, u) \geq \gamma\|u\|^{2}, & \forall u \in \mathcal{V} \\
l_{\varepsilon}(v) & \leq L\|v\|,
\end{array}
$$

Assume that there exist a bilinear and continuous form $\delta a$, a linear and continuous form $\delta l$, and a real function $\rho(\varepsilon)>0$ defined on $\mathbb{R}_{+}$such that

$$
\begin{gathered}
\left\|a_{\varepsilon}-a_{0}-\rho(\varepsilon) \delta a\right\|_{\mathcal{L}_{2}(\mathcal{V})}=o(\rho(\varepsilon)), \\
\left\|l_{\varepsilon}-l_{0}-\rho(\varepsilon) \delta l\right\|_{\mathcal{L}(\mathcal{V})}=o(\rho(\varepsilon)), \\
\lim _{\varepsilon \rightarrow 0} \rho(\varepsilon)=0
\end{gathered}
$$

where $\mathcal{L}(\mathcal{V})$ (respectively $\mathcal{L}_{2}(\mathcal{V})$ ) denotes the space of continuous and linear (respectively bilinear) forms on $\mathcal{V}$. For $\varepsilon \geq 0$, let $u_{\varepsilon}$ be the solution to the problem: find $u_{\varepsilon} \in \mathcal{V}$ such that

$$
a_{\varepsilon}\left(u_{\varepsilon}, v\right)=l_{\varepsilon}(v), \quad \forall v \in \mathcal{V} .
$$

Lemma 2.1 [15]. For $\varepsilon \geq 0$, problem (3) has a unique solution $u_{\varepsilon}$, and

$$
\left\|u_{\varepsilon}-u_{0}\right\|=O(\rho(\varepsilon))
$$

Next we consider a cost function of the form $j(\varepsilon)=J_{\varepsilon}\left(u_{\varepsilon}\right)$, where $J_{\varepsilon}$ is defined on $\mathcal{V}$ for $\varepsilon \geq 0$ and $J_{0}$ is differentiable with respect to $u$, its derivative being denoted by $D J_{0}(u)$.

Suppose that there exists a function $\delta J$ defined on $\mathcal{V}$ such that: for all $\varepsilon>0$

$$
J_{\varepsilon}(v)-J_{0}(u)=D J_{0}(u)(v-u)+\rho(\varepsilon) \delta J(u)+o(\|v-u\|+\rho(\varepsilon)) \quad \forall u, v \in \mathcal{V} .
$$

Theorem 2.1 [15,29]. Under the hypotheses (1), (2) and (5) the function $j$ has the following asymptotic expansion

$$
j(\varepsilon)=j(0)+\rho(\varepsilon)\left[\delta a\left(u_{0}, v_{0}\right)-\delta l\left(v_{0}\right)+\delta J\left(u_{0}\right)\right]+o(\rho(\varepsilon))
$$

where $v_{0} \in \mathcal{V}$ is the solution to the adjoint problem: find $v_{0} \in \mathcal{V}$ such that

$$
a_{0}\left(w, v_{0}\right)=-D J_{0}\left(u_{0}\right) w, \quad \forall w \in \mathcal{V} \text {. }
$$

\section{Position of the problem}

\subsection{The Quasi-Stokes equations}

We consider $\Omega$ a bounded domain of $\mathbb{R}^{N}, N=2,3$. We denote by $\Gamma$ its boundary.

The standard form of the Navier-Stokes equations describing the motion of an incompressible fluid in $\Omega$ is given by:

$$
\left\{\begin{aligned}
\frac{\partial u_{\Omega}}{\partial t}+\left(u_{\Omega} . \nabla\right) u_{\Omega}-\nu \Delta u_{\Omega}+\nabla p_{\Omega} & =F & & \text { in } \Omega \\
\nabla . u_{\Omega} & =0 & & \text { in } \Omega \\
u_{\Omega} & =\mathcal{U}_{d} & & \text { on } \Gamma
\end{aligned}\right.
$$

where $u_{\Omega}$ and $p_{\Omega}$ denote respectively the velocity and the pressure fields, $F$ is a given body force per unit of mass, $\nu$ denotes the kinematic viscosity of the fluid and $\mathcal{U}_{d}$ is a given boundary velocity. 
Because of the divergence-free condition on $u_{\Omega}, \mathcal{U}_{d}$ must necessary satisfy the compatibility condition

$$
\int_{\Gamma} \mathcal{U}_{d} \cdot n \mathrm{~d} s=0
$$

where $n$ is the unit normal vector along the boundary $\Gamma$.

Denoting by $\Delta t$ the time step and posing $t^{n}=n \Delta t$ and $t^{n+1}=t^{n}+\Delta t$.

Using the characteristic method (see, e.g., [12]), an approximation for the convection term is given by

$$
\left.\left(\frac{\partial u_{\Omega}}{\partial t}(t, x)+u_{\Omega} \cdot \nabla u_{\Omega}(t, x)\right)\right|_{\left(t=t^{n}\right)}=\left.\frac{\mathrm{d} u_{\Omega}}{\mathrm{d} t}(t, x(t))\right|_{\left(t=t^{n}\right)} \simeq \frac{u_{\Omega}\left(t^{n+1}, x\left(t^{n+1}\right)\right)-u_{\Omega}\left(t^{n}, x\left(t^{n}\right)\right)}{\Delta t} .
$$

By an implicit scheme, a time discretization of the system (7) can be written as

$$
\left\{\begin{aligned}
\frac{1}{\Delta t} u_{\Omega}^{n+1}-\nu \Delta u_{\Omega}^{n+1}+\nabla p_{\Omega}^{n+1} & =F^{n+1}+\frac{u_{\Omega}\left(t^{n}, x\left(t^{n}\right)\right)}{\Delta t} \\
\nabla \cdot u^{n+1} & =0 .
\end{aligned}\right.
$$

Then, at each time step, we have to solve a steady state Quasi-Stokes problem, called also generalized Stokes problem, having the following generic form

$$
\left\{\begin{aligned}
\alpha u_{\Omega}-\nu \Delta u_{\Omega}+\nabla p_{\Omega} & =f & & \text { in } \Omega \\
\nabla \cdot u_{\Omega} & =0 & & \text { in } \Omega \\
u_{\Omega} & =\mathcal{U}_{d} & & \text { on } \Gamma .
\end{aligned}\right.
$$

\subsection{The shape optimization problem}

For a given $x_{0} \in \Omega$, consider the modified domain $\Omega_{\varepsilon}=\Omega \backslash \bar{\omega}_{\varepsilon}, \omega_{\varepsilon}=x_{0}+\varepsilon \omega$, where $\omega$ is a given fixed and bounded domain of $\mathbb{R}^{N}$, containing the origin, whose boundary $\partial \omega$ is connected and piecewise of class $\mathcal{C}^{1}$.
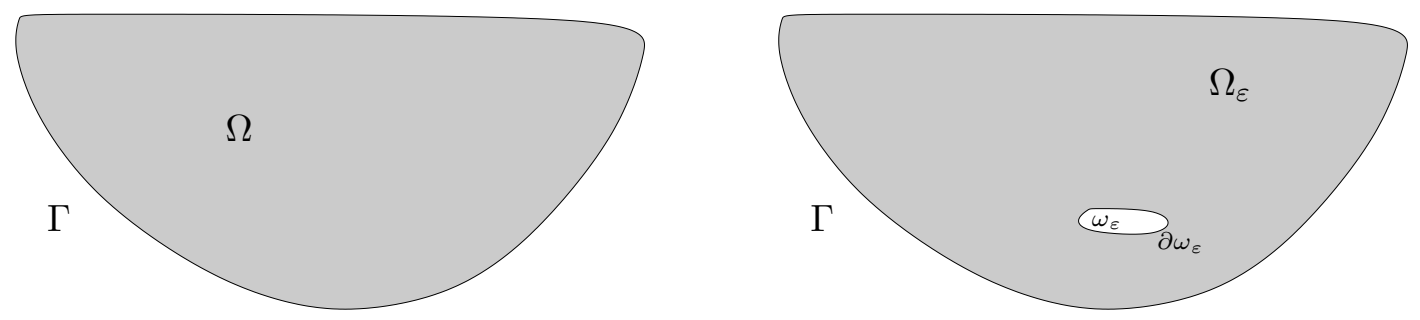

Figure 1. The initial domain and the same domain after creation of a small hole $\omega_{\varepsilon}$.

In the modified domain $\Omega_{\varepsilon}$, the velocity and pressure fields are required to satisfy

$$
\left\{\begin{aligned}
\alpha u_{\Omega_{\varepsilon}}-\nu \Delta u_{\Omega_{\varepsilon}}+\nabla p_{\Omega_{\varepsilon}} & =f & & \text { in } \Omega_{\varepsilon} \\
\nabla . u_{\Omega_{\varepsilon}} & =0 & & \text { in } \Omega_{\varepsilon} \\
u_{\Omega_{\varepsilon}} & =\mathcal{U}_{d} & & \text { on } \Gamma \\
u_{\Omega_{\varepsilon}} & =\mathcal{U}_{i n j} & & \text { on } \partial \omega_{\varepsilon}
\end{aligned}\right.
$$

where $\mathcal{U}_{i n j}$ is a given velocity on $\partial \omega_{\varepsilon}$.

Note that for $\varepsilon=0$, one has $u_{\Omega_{0}}=u_{\Omega}$ and $p_{\Omega_{0}}=p_{\Omega}$. 
Theorem 3.1 $[4,17,38]$. Let $\varepsilon \geq 0$, for a given $f \in L^{2}\left(\Omega_{\varepsilon}\right)^{N}, \mathcal{U}_{i n j} \in H^{1 / 2}\left(\partial \omega_{\varepsilon}\right)^{N}$ and $\mathcal{U}_{d} \in H^{1 / 2}(\Gamma)^{N}$, the problem (9) has a unique solution $\left(u_{\Omega_{\varepsilon}}, p_{\Omega_{\varepsilon}}\right) \in H^{1}\left(\Omega_{\varepsilon}\right)^{N} \times L_{0}^{2}\left(\Omega_{\varepsilon}\right)^{N}$ where $L_{0}^{2}\left(\Omega_{\varepsilon}\right)^{N}=\left\{\theta \in L^{2}\left(\Omega_{\varepsilon}\right)^{N}, \int_{\Omega_{\varepsilon}} \theta \mathrm{d} x=0\right\}$.

Let $\bar{u}_{\Omega_{\varepsilon}}$ an extension of the boundary data $\mathcal{U}_{d}$ and $\mathcal{U}_{i n j}$ in $\Omega_{\varepsilon},\left(\partial \Omega_{\varepsilon}=\Gamma \cup \partial \omega_{\varepsilon}\right)$, satisfying

$$
\left\{\begin{aligned}
\alpha \bar{u}_{\Omega_{\varepsilon}}-\nu \Delta \bar{u}_{\Omega_{\varepsilon}}+\nabla \bar{p}_{\Omega_{\varepsilon}} & =0 & & \text { in } \Omega_{\varepsilon} \\
\nabla \cdot \bar{u}_{\Omega_{\varepsilon}} & =0 & & \text { in } \Omega_{\varepsilon} \\
\bar{u}_{\Omega_{\varepsilon}} & =\mathcal{U}_{d} & & \text { on } \Gamma \\
\bar{u}_{\Omega_{\varepsilon}} & =\mathcal{U}_{i n j} & & \text { on } \partial \omega_{\varepsilon} .
\end{aligned}\right.
$$

The solution $u_{\Omega_{\varepsilon}}$ can be recuperated as $u_{\Omega_{\varepsilon}}=w_{\Omega_{\varepsilon}}+\bar{u}_{\Omega_{\varepsilon}}$, with $w_{\Omega_{\varepsilon}}$ is the solution of the system (9) with a homogeneous Dirichlet boundary condition on $\partial \Omega_{\varepsilon}$.

Thanks to the previous variable substitution, in the theoretical part of this work, we will consider only a homogeneous boundary condition. Then, we will assume that $\mathcal{U}_{d}=0$ on $\Gamma$ and $\mathcal{U}_{i n j}=0$ on $\partial \omega_{\varepsilon}$.

We now consider a cost function $j(\varepsilon)$ of the form

$$
j(\varepsilon)=\widetilde{J}_{\varepsilon}\left(u_{\Omega_{\varepsilon}}\right),
$$

with $\widetilde{J}_{\varepsilon}$ being defined on $H^{1}\left(\Omega_{\varepsilon}\right)^{N}$ for $\varepsilon \geq 0$.

Our aim is to obtain an asymptotic expansion of $j$ with respect to $\varepsilon$. The velocity field $u_{\Omega_{\varepsilon}}$ is defined in the variable domain $\Omega_{\varepsilon}$, thus it belongs to a functional space which depends on $\varepsilon$. Hence, if we want to derive the asymptotic expansion of $j$ we cannot apply directly the results of Section 2, which require a fixed functional space (cf. Th. 2.1).

In classical shape optimization, this condition is satisfied by the mean of a domain parameterization method. This method involves a fixed domain and a bi-Lipshitz map between the initial domain and the modified one. In the topology optimization context, such a map does not exist between $\Omega$ and $\Omega_{\varepsilon}$.

However, a functional space independent of $\varepsilon$ can be constructed by using a domain truncation technique described in the next paragraph (see also [29] and [15]).

This truncation is needed only for analysis, and will never be used for practical computation. During the optimization process, we have just to solve the system (8) and the adjoint problem associated to the cost function (11).

\section{The TRUnCATED PROBLEM}

Let $R>0$ be such that the closed ball $\overline{B\left(x_{0}, R\right)}$ is included in $\Omega$ and $\bar{\omega}_{\varepsilon} \subset B\left(x_{0}, R\right)$.
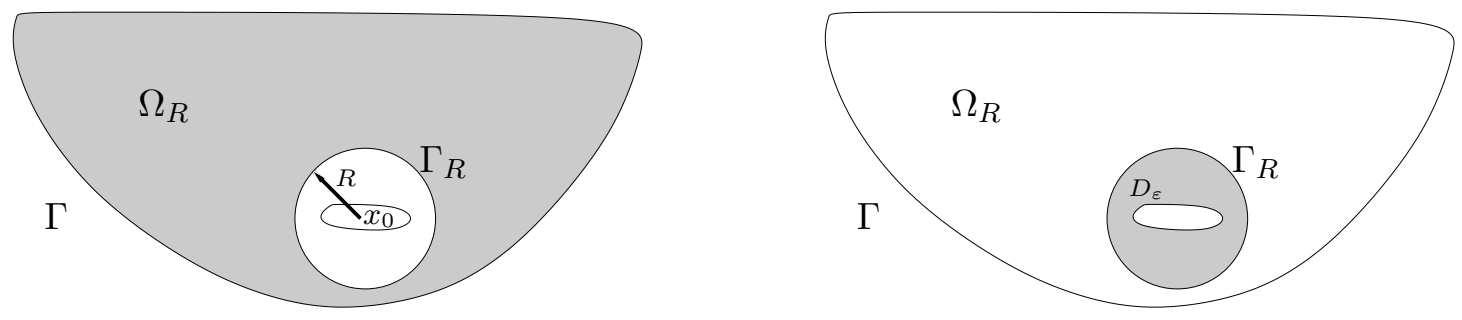

Figure 2. The truncated domain. 
We denote by $\Gamma_{R}$ the boundary of $B\left(x_{0}, R\right)$ and we consider the fixed domain $\Omega_{R}=\Omega \backslash \overline{B\left(x_{0}, R\right)}$ and $D_{\varepsilon}=B\left(x_{0}, R\right) \backslash \overline{\omega_{\varepsilon}}$. Also we use the following space of traces on $\Gamma_{R}$

$$
H_{V}^{1 / 2}\left(\Gamma_{R}\right)^{N}=\left\{\phi \in H^{1 / 2}\left(\Gamma_{R}\right)^{N} ; \quad \int_{\Gamma_{R}} \phi . n \mathrm{~d} x=0\right\}
$$

where $n$ is the unit vector normal to $\Gamma_{R}$. Its dual space is denoted by $H_{V}^{-1 / 2}\left(\Gamma_{R}\right)^{N}$.

For a given $f \in L^{2}(\Omega)^{N}, \varphi \in H_{V}^{1 / 2}\left(\Gamma_{R}\right)^{N}$ and $\varepsilon>0$, let $u_{\varepsilon}^{f, \varphi}, p_{\varepsilon}^{f, \varphi}$ be the solution to the problem: find $\left(u_{\varepsilon}^{f, \varphi}, p_{\varepsilon}^{f, \varphi}\right) \in H^{1}\left(D_{\varepsilon}\right)^{N} \times L_{0}^{2}\left(D_{\varepsilon}\right)^{N}$ such that

$$
\left\{\begin{aligned}
\alpha u_{\varepsilon}^{f, \varphi}-\nu \Delta u_{\varepsilon}^{f, \varphi}+\nabla p_{\varepsilon}^{f, \varphi}=f & \text { in } D_{\varepsilon} \\
\operatorname{div} u_{\varepsilon}^{f, \varphi}=0 & \text { in } D_{\varepsilon} \\
u_{\varepsilon}^{f, \varphi}=\varphi & \text { on } \Gamma_{R} \\
u_{\varepsilon}^{f, \varphi}=0 & \text { on } \partial \omega_{\varepsilon} .
\end{aligned}\right.
$$

This problem has a unique solution $[4,17,38]$.

For $\varepsilon=0,\left(u_{0}^{f, \varphi}, p_{0}^{f, \varphi}\right)$ is the solution to

$$
\left\{\begin{aligned}
\alpha u_{0}^{f, \varphi}-\nu \Delta u_{0}^{f, \varphi}+\nabla p_{0}^{f, \varphi}=f & & \text { in } B\left(x_{0}, R\right) \\
\operatorname{div} u_{0}^{f, \varphi}=0 & & \text { in } B\left(x_{0}, R\right) \\
u_{0}^{f, \varphi}=\varphi & & \text { on } \Gamma_{R} .
\end{aligned}\right.
$$

Clearly we have

$$
u_{\varepsilon}^{f, \varphi}=u_{\varepsilon}^{f, 0}+u_{\varepsilon}^{0, \varphi}, \quad p_{\varepsilon}^{f, \varphi}=p_{\varepsilon}^{f, 0}+p_{\varepsilon}^{0, \varphi}, \quad \forall \varepsilon \geq 0 .
$$

This decomposition will be used to construct the bilinear form $a_{\varepsilon}$ and the linear form $l_{\varepsilon}$ presented in Section 2 . For $\varepsilon \geq 0$, we consider the Dirichlet-to-Neumann operator

$$
\begin{aligned}
T_{\varepsilon}: H_{V}^{1 / 2}\left(\Gamma_{R}\right)^{N} & \longrightarrow H_{V}^{-1 / 2}\left(\Gamma_{R}\right)^{N} \\
\varphi & \longmapsto T_{\varepsilon} \varphi=\sigma\left(u_{\varepsilon}^{0, \varphi}\right) . n
\end{aligned}
$$

where $\sigma\left(u_{\varepsilon}^{0, \varphi}\right)=\left(\nu \nabla u_{\varepsilon}^{0, \varphi}-p_{\varepsilon}^{0, \varphi} I\right)$ is the stress tensor.

And the function $f_{\varepsilon} \in H_{V}^{-1 / 2}\left(\Gamma_{R}\right)^{N}$

$$
f_{\varepsilon}=-\sigma\left(u_{\varepsilon}^{f, 0}\right) \cdot n=-\left(\nu \nabla u_{\varepsilon}^{f, 0}-p_{\varepsilon}^{f, 0} I\right) \cdot n
$$

with the normal $n$ is chosen outward to $D_{\varepsilon}$ on $\Gamma_{R}$ and $\partial \omega_{\varepsilon}$.

Hence, for all $\varepsilon \geq 0$ and $\varphi \in H_{V}^{1 / 2}\left(\Gamma_{R}\right)^{N}$ we have $\sigma\left(u_{\varepsilon}^{f, \varphi}\right) . n=T_{\varepsilon} \varphi-f_{\varepsilon}$.

Finally, we define for $\varepsilon \geq 0$ the solution $u_{\varepsilon}, p_{\varepsilon}$ to the truncated problem

$$
\left\{\begin{aligned}
\alpha u_{\varepsilon}-\nu \Delta u_{\varepsilon}+\nabla p_{\varepsilon}=f & \text { in } \Omega_{R} \\
\operatorname{div} u_{\varepsilon}=0 & \text { in } \Omega_{R} \\
u_{\varepsilon}=0 & \text { on } \Gamma \\
\sigma\left(u_{\varepsilon}\right) \cdot n+T_{\varepsilon} u_{\varepsilon}=f_{\varepsilon} & \text { on } \Gamma_{R}
\end{aligned}\right.
$$

The variational formulation associated to (18) is: find $u_{\varepsilon} \in \mathcal{V}_{R}$ such that

$$
a_{\varepsilon}\left(u_{\varepsilon}, v\right)=l_{\varepsilon}(v), \quad \forall v \in \mathcal{V}_{R}
$$


where the functional space $\mathcal{V}_{R}$, the bilinear form $a_{\varepsilon}$ and the linear form $l_{\varepsilon}$ are defined by

$$
\begin{gathered}
\mathcal{V}_{R}=\left\{u \in H^{1}\left(\Omega_{R}\right)^{N} ; \operatorname{div} u=0, u=0 \text { on } \Gamma\right\}, \\
a_{\varepsilon}(u, v)=\alpha \int_{\Omega_{R}} u . v \mathrm{~d} x+\nu \int_{\Omega_{R}} \nabla u: \nabla v \mathrm{~d} x+\int_{\Gamma_{R}} T_{\varepsilon} u v \mathrm{~d} \gamma(x), \\
l_{\varepsilon}(v)=\int_{\Omega_{R}} f v \mathrm{~d} x+\int_{\Gamma_{R}} f_{\varepsilon} v \mathrm{~d} \gamma(x) .
\end{gathered}
$$

Symmetry, continuity and coercivity of $a_{\varepsilon}$ and continuity of $l_{\varepsilon}$ follow directly from

$$
\begin{gathered}
\int_{\Gamma_{R}} T_{\varepsilon} \varphi \psi \mathrm{d} \gamma(x)=\alpha \int_{D_{\varepsilon}} u_{\varepsilon}^{0, \varphi} \cdot u_{\varepsilon}^{0, \psi} \mathrm{d} x+\nu \int_{D_{\varepsilon}} \nabla u_{\varepsilon}^{0, \varphi}: \nabla u_{\varepsilon}^{0, \psi} \mathrm{d} x, \\
\int_{\Gamma_{R}} f_{\varepsilon} \psi \mathrm{d} \gamma(x)=\int_{D_{\varepsilon}} f u_{\varepsilon}^{0, \psi} \mathrm{d} x,
\end{gathered}
$$

and the relation

$$
\alpha \int_{D_{\varepsilon}} u_{\varepsilon}^{f, 0} \cdot u_{\varepsilon}^{0, \psi} \mathrm{d} x+\nu \int_{D_{\varepsilon}} \nabla u_{\varepsilon}^{f, 0}: \nabla u_{\varepsilon}^{0, \psi} \mathrm{d} x=0 .
$$

Proposition 4.1. Let $\varepsilon \geq 0$. Problems (9) and (18) have unique solution.

Moreover, the restriction to $\Omega_{R}$ of the solution $u_{\Omega_{\varepsilon}}, p_{\Omega_{\varepsilon}}$ to (9) is the solution $u_{\varepsilon}, p_{\varepsilon}$ to (18), and we have in $D_{\varepsilon}$

$$
\left(u_{\Omega_{\varepsilon}}\right)_{\mid D_{\varepsilon}}=u_{\varepsilon}^{f, \varphi}, \quad\left(p_{\Omega_{\varepsilon}}\right)_{\mid D_{\varepsilon}}=p_{\varepsilon}^{f, \varphi}
$$

where $\varphi$ is the trace of $u_{\Omega_{\varepsilon}}$ on $\Gamma_{R}$.

Proof. We refer to $[4,17,38]$ for the existence and uniqueness of the solutions to both problems (9) and (18). Recall that we have denoted by $\left(u_{\Omega_{\varepsilon}}, p_{\Omega_{\varepsilon}}\right)$ the solution of $(9)$ and by $\left(u_{\varepsilon}, p_{\varepsilon}\right)$ the solution of (18).

Let $\varphi=u_{\Omega_{\varepsilon} \mid \Gamma_{R}}$ and $u_{R}=u_{\Omega_{\varepsilon} \mid \Omega_{R}}$. Clearly (25) holds for this $\varphi$, and it remains to prove that $u_{R}=u_{\varepsilon}$.

Let $\theta \in \mathcal{V}_{R}$ and $\psi=\theta_{\mid \Gamma_{R}}$. We extend $\theta$ on $D_{\varepsilon}$ by $u_{\varepsilon}^{0, \psi}$. Its extension is still denoted by $\theta$, and it is divergence free on $D_{\varepsilon}$.

Using (22), (23), (24) and the definition of $u_{\Omega_{\varepsilon}}$, we have

$$
\begin{aligned}
\alpha & \int_{\Omega_{R}} u_{R} \cdot \theta \mathrm{d} x+\nu \int_{\Omega_{R}} \nabla u_{R}: \nabla \theta \mathrm{d} x+\int_{\Gamma_{R}}\left(T_{\varepsilon} u_{R}-f_{\varepsilon}\right) \theta \mathrm{d} \gamma(x) \\
= & \alpha \int_{\Omega_{R}} u_{R} \cdot \theta \mathrm{d} x+\nu \int_{\Omega_{R}} \nabla u_{R}: \nabla \theta \mathrm{d} x+\int_{\Gamma_{R}} T_{\varepsilon} u_{R} \theta \mathrm{d} \gamma(x)-\int_{\Gamma_{R}} f_{\varepsilon} \cdot \theta \mathrm{d} \gamma(x) \\
= & \alpha \int_{\Omega_{R}} u_{R} \cdot \theta \mathrm{d} x+\nu \int_{\Omega_{R}} \nabla u_{R}: \nabla \theta \mathrm{d} x+\alpha \int_{D_{\varepsilon}} u_{\varepsilon}^{0, \varphi} \cdot u_{\varepsilon}^{0, \psi} \mathrm{d} x \\
& +\nu \int_{D_{\varepsilon}} \nabla u_{\varepsilon}^{0, \varphi}: \nabla u_{\varepsilon}^{0, \psi} \mathrm{d} x-\int_{D_{\varepsilon}} f \cdot u_{\varepsilon}^{0, \psi} \mathrm{d} x \\
= & \alpha \int_{\Omega_{\varepsilon}} u_{\varepsilon} \cdot \theta \mathrm{d} x+\nu \int_{\Omega_{\varepsilon}} \nabla u_{\varepsilon}: \nabla \theta \mathrm{d} x-\int_{\Gamma_{R}} f \cdot u_{\varepsilon}^{0, \psi} \mathrm{d} \gamma(x) \\
= & \alpha \int_{\Omega_{\varepsilon}}^{u_{\varepsilon}} \cdot \theta \mathrm{d} x+\nu \int_{\Omega_{\varepsilon}} \nabla u_{\varepsilon}: \nabla \theta \mathrm{d} x-\int_{D_{\varepsilon}} f \cdot \theta \mathrm{d} x=\int_{\Omega_{R}} f \cdot \theta \mathrm{d} x .
\end{aligned}
$$

This proves that $u_{R}$ is the solution to (19). From uniqueness of the solution, we deduce that $u_{R}=u_{\varepsilon}$.

Now we have at our disposal the fixed Hilbert space $\mathcal{V}_{R}$ required by Section 2. The cost function (11) can be redefined in the following way: 
for $u \in \mathcal{V}_{R}$, let $\varphi=u_{\mid \Gamma_{R}}$, one defines $\tilde{u}_{\varepsilon} \in H^{1}\left(\Omega_{\varepsilon}\right)^{N}$ the extension of $u$ on $\Omega_{\varepsilon}$ as follow

$$
\tilde{u}_{\varepsilon}= \begin{cases}u & \text { on } \Omega_{R} \\ u_{\varepsilon}^{f, \varphi} & \text { on } D_{\varepsilon}\end{cases}
$$

Then, a function $J_{\varepsilon}$ can be defined on $\mathcal{V}_{R}$ by

$$
J_{\varepsilon}(u)=\tilde{J}_{\varepsilon}\left(\tilde{u}_{\varepsilon}\right)
$$

Particularly, we have from the previous proposition that

$$
j(\varepsilon)=\tilde{J}_{\varepsilon}\left(u_{\Omega_{\varepsilon}}\right)=J_{\varepsilon}\left(u_{\varepsilon}\right) .
$$

Remark that $J_{\varepsilon}\left(u_{\varepsilon}\right)$ is independent of the choice of $R$. For example, for a given target function $\mathcal{U}_{g}$, if

$$
\tilde{J}_{\varepsilon}\left(u_{\Omega_{\varepsilon}}\right)=\int_{\Omega_{\varepsilon}}\left|u_{\Omega_{\varepsilon}}-\mathcal{U}_{g}\right|^{2} \mathrm{~d} x
$$

then, we have for all $u \in \mathcal{V}_{R}$

$$
J_{\varepsilon}(u)=\int_{\Omega_{R}}\left|u-\mathcal{U}_{g}\right|^{2} \mathrm{~d} x+\int_{D_{\varepsilon}}\left|u_{\varepsilon}^{f, \varphi}-\mathcal{U}_{g}\right|^{2} \mathrm{~d} x, \text { with } \varphi=u_{\mid \Gamma_{R}} .
$$

\section{MAIN RESUlT: THE ASYMPTOTIC EXPANSION}

In this section, we present the main results of this paper, which concern the asymptotic analysis with respect to the parameter $\varepsilon$ of the functional (27). An asymptotic expansion is obtained for the Quasi-Stokes operator for a large class of cost functions and arbitrary shaped holes.

We begin by the three dimensional case. The principal result is given by Theorem 5.1, it gives the topological sensitivity expression $\delta j\left(x_{0}\right)$ if a hole is created at $x_{0}$. The proof of Theorem 5.1 is relegated to Section 7 .

\subsection{The three dimensional case}

In order to derive the topological sensitivity of the function $j$, we introduce two auxiliary problems.

The first problem, which we call the "exterior problem", is formulated in $\mathbb{R}^{3} \backslash \bar{\omega}$ and consists to find $(U, P)$ solution to

$$
\left\{\begin{aligned}
-\nu \Delta U+\nabla P & =0 & & \text { in } \mathbb{R}^{3} \backslash \bar{\omega} \\
\operatorname{div} U & =0 & & \text { in } \mathbb{R}^{3} \backslash \bar{\omega} \\
U & =0 & & \text { at infinity } \\
U & =u_{\Omega}\left(x_{0}\right) & & \text { on } \partial \omega
\end{aligned}\right.
$$

where $u_{\Omega}$ is the solution to Quasi-Stokes problem (8). Recall that $f \in L^{2}(\Omega)^{3}$, so that $u_{\Omega}$ is continuous inside $\Omega$.

Here, one can remark that just the principal part of the Quasi-Stokes operator is used, which is the Stokes equations. A such approach can be justified by the fact that operators $-\Delta$ and $I-\Delta$ have the same behavior near the hole $\omega_{\varepsilon}$ and give the same leading terms for topological expansion. Moreover, this approach has a good advantage, it avoids calculations with the complicated expression of the Quasi-Stokes fundamental solution, which involves the convolution product of Green functions for both operators $I-\Delta$ and $\Delta$.

We return now to the system (31). The functions $U, P$ can be expressed by a simple layer potential on $\partial \omega$. Coordinate system can be changed, in what follows one can suppose for convenience that $x_{0}=0$.

Posing $r=\|y\|$ and $e_{r}=\frac{y}{\|y\|}$, the fundamental solution system to the Stokes equations in $\mathbb{R}^{3}$ can be written as

$$
G_{U}(y)=\frac{1}{8 \pi \nu r}\left(I+e_{r} e_{r}^{T}\right), \quad G_{P}(y)=\frac{y}{4 \pi r^{3}}
$$


such that

$$
-\nu \Delta G_{U j}+\nabla G_{P j}=\delta e_{j}
$$

where $G_{U j}$ denote the $j$ th column of $G_{U},\left\{e_{j}\right\}_{j=1,3}$ is the canonical basis of $\mathbb{R}^{3}$ and $\delta$ is the Dirac distribution. Then, the functions $U, P$ read [11]

$$
\begin{aligned}
& U(y)=\int_{\partial \omega} G_{U}(y-x) T(x) \mathrm{d} \gamma(x), \quad y \in \mathbb{R}^{3} \backslash \bar{\omega} \\
& P(y)=\int_{\partial \omega} G_{P}(y-x) \cdot T(x) \mathrm{d} \gamma(x), \quad y \in \mathbb{R}^{3} \backslash \bar{\omega}
\end{aligned}
$$

where $T \in H^{-1 / 2}(\partial \omega)^{3}$ is a solution to the boundary integral equation (see e.g. [11])

$$
\int_{\partial \omega} G_{U}(y-x) T(x) \mathrm{d} \gamma(x)=u_{\Omega}\left(x_{0}\right), \quad y \in \partial \omega .
$$

One can observe that the function $T$ is determined up to a function proportional to the normal, hence it is unique in $H^{-1 / 2}(\partial \omega)^{3} / \mathbb{R} n$.

Using the first order Taylor expansion of $G_{U}$ at the point $y \neq 0$ for $x$ bounded, we have

$$
G_{U}(y-x)=G_{U}(y)+O\left(\frac{1}{r^{2}}\right), \quad G_{P}(y-x)=G_{P}(y)+O\left(\frac{1}{r^{3}}\right) ;
$$

from which follows the asymptotic expansion at infinity of $U$ and $P$ :

$$
U(y)=S_{U}(y)+L_{U}(y), \quad P(y)=S_{P}(y)+L_{P}(y)
$$

where $S_{U}(y)$ and $S_{P}(y)$ are the dominant part respectively of $U$ and $P$

$$
S_{U}(y)=G_{U}(y) A\left(u_{\Omega}\left(x_{0}\right)\right), \quad S_{P}(y)=G_{P}(y) \cdot A\left(u_{\Omega}\left(x_{0}\right)\right)
$$

with

$$
A\left(u_{\Omega}\left(x_{0}\right)\right)=\int_{\partial \omega} T(x) \mathrm{d} \gamma(x) \in \mathbb{R}^{3} .
$$

Notice that $\alpha \longrightarrow A(\alpha)$ is linear on $\mathbb{R}^{3}$ and $S_{U} \in L_{\text {loc }}^{2}\left(\mathbb{R}^{3}\right)$.

The last parts of $U$ and $P$ are respectively given by

$$
L_{U}(y)=O\left(\frac{1}{r^{2}}\right), \quad L_{P}(y)=O\left(\frac{1}{r^{3}}\right) .
$$

The second problem, which we call "interior problem", is formulated in $D_{0}=B\left(x_{0}, R\right)$ and consists to find $\left(R_{U}^{h}, R_{P}^{h}\right)$ solution to

$$
\left\{\begin{aligned}
\alpha R_{U}^{h}-\nu \Delta R_{U}^{h}+\nabla R_{P}^{h}=0 & & \text { in } B\left(x_{0}, R\right) \\
\operatorname{div} R_{U}^{h}=0 & & \text { in } B\left(x_{0}, R\right) \\
R_{U}^{h}=S_{U} & & \text { on } \Gamma_{R} .
\end{aligned}\right.
$$

Here, the idea is to consider an interior and exterior problems that gives the asymptotic behavior of $\left(u_{\varepsilon}^{f, \varphi}\right.$ $\left.-u_{0}^{f, \varphi}\right)\left.\right|_{D_{\varepsilon}}$ with $\varphi=\left.u_{\Omega}\right|_{\Gamma_{R}}$ in a sense which will be stated in Section 7 .

It will not be possible to derive the asymptotic behavior of $u_{\varepsilon}^{f, \varphi}-u_{0}^{f, \varphi}$ from $R_{U}^{h}-S_{U}$. We have first to take into account the error due to the simplification of the fundamental solution. We propose to cancel this error by adding a correcting term to $R_{U}^{h}$. 
In such a case, we consider the correcting term $\left(R_{U}^{c}, R_{P}^{c}\right)$ as solution to

$$
\left\{\begin{aligned}
\alpha R_{U}^{c}-\nu \Delta R_{U}^{c}+\nabla R_{P}^{c}=\alpha S_{U} & \text { in } B\left(x_{0}, R\right) \\
\operatorname{div} R_{U}^{c}=0 & \text { in } B\left(x_{0}, R\right) \\
R_{U}^{c}=0 & \text { on } \Gamma_{R}
\end{aligned}\right.
$$

Setting $R_{U}=R_{U}^{h}+R_{U}^{c}, R_{P}=R_{P}^{h}+R_{P}^{c}$, then $\left(R_{U}, R_{P}\right)$ is solution to

$$
\left\{\begin{array}{rlrl}
\alpha R_{U}-\nu \Delta R_{U}+\nabla R_{P} & =\alpha S_{U} & \text { in } B\left(x_{0}, R\right) \\
\operatorname{div} R_{U} & =0 & \text { in } B\left(x_{0}, R\right) \\
R_{U} & =S_{U} & & \text { on } \Gamma_{R}
\end{array}\right.
$$

We will prove in Section 7, using the corrected interior problem (43), it will be possible to derive the asymptotic behavior of $\left(u_{\varepsilon}^{f, \varphi}-u_{0}^{f, \varphi}\right)_{\mid D_{\varepsilon}}$. The main result is the following. It will be proved in Section 7 .

Theorem 5.1. Let $f \in H^{2}(\Omega)^{3}$, and let $j(\varepsilon)=J_{\varepsilon}\left(u_{\varepsilon}\right)$. Suppose that $J_{\varepsilon}$ satisfy the hypothesis (5): for all $v \in \mathcal{V}_{R}$ and all $\varepsilon>0$

$$
J_{\varepsilon}(v)-J_{0}\left(u_{0}\right)=D J_{0}\left(u_{0}\right)\left(v-u_{0}\right)+\varepsilon \delta J\left(u_{0}\right)+o\left(\varepsilon+\left\|v-u_{0}\right\|_{\mathcal{V}_{R}}\right),
$$

where $D J_{0}\left(u_{0}\right)$ is linear and continuous on $\mathcal{V}_{R}$, and $u_{\varepsilon}, \varepsilon \geq 0$ is the solution to (19).

Let $v_{0} \in \mathcal{V}_{R}$ be the solution to the adjoint equation

$$
a_{0}\left(w, v_{0}\right)=-D J_{0}\left(u_{0}\right) w, \quad \forall w \in \mathcal{V}_{R}
$$

Then, the function $j$ has the following asymptotic expansion

$$
j(\varepsilon)=j(0)+\varepsilon \delta j\left(x_{0}\right)+o(\varepsilon)
$$

with

$$
\delta j\left(x_{0}\right)=\int_{\Gamma_{R}}\left(\sigma\left(R_{U}-S_{U}\right)\right) \cdot n v_{0} \mathrm{~d} \gamma(x)+\delta J\left(u_{0}\right) .
$$

The functional $\delta j\left(x_{0}\right)$ is called the "topological sensitivity" of the Quasi-Stokes operator. It is also called the "topological gradient".

The cost function $j$ is independent of $R$ and $\delta j\left(x_{0}\right)$ is independent of $\varepsilon$, then $\delta j\left(x_{0}\right)$ is also independent of $R$. This follows from the uniqueness of an asymptotic expansion. As we will observe in Section 7 , this is not necessarily true for the terms $\delta a\left(u_{0}, v_{0}\right), \delta l\left(v_{0}\right)$ or $\delta J\left(u_{0}\right)$, because $a, l$ and $J$ do depend on $R$.

Practically, we need just to compute the solution $u_{\Omega}$ to (8) and $v_{\Omega}$ the solution to the associated adjoint problem

$$
\alpha \int_{\Omega} w v_{\Omega} \mathrm{d} x+\nu \int_{\Omega} \nabla w: \nabla v_{\Omega} \mathrm{d} x=-D \tilde{J}_{0}\left(u_{\Omega}\right) w, \quad \forall w \in \mathcal{V}_{0}
$$

with $\mathcal{V}_{0}=\left\{v \in H_{0}^{1}(\Omega)^{3} ; \operatorname{div} v=0\right.$ in $\left.\Omega\right\}$.

It has been shown in Proposition 4.1 that $u_{0}$ is the restriction to $\Omega_{R}$ of $u_{\Omega}$. Similarly, $v_{0}$ is the restriction to $\Omega_{R}$ of $v_{\Omega}$, this can be proved in the same way. Consequently, the function $u_{\Omega}$ (or $u_{0}$ ) and the adjoint state $v_{\Omega}$ ( or $v_{0}$ ) do not depend on $x_{0}$. Hence, the basic property of an adjoint technique is here satisfied: only two systems have to be solved in order to compute the topological sensitivity $\delta j(x)$ for all $x \in \Omega$.

Moreover, there exists a unique $q_{\Omega} \in L_{0}^{2}(\Omega)^{3}$ such that

$$
\alpha \int_{\Omega} w v_{\Omega} \mathrm{d} x+\nu \int_{\Omega} \nabla w: \nabla v_{\Omega} \mathrm{d} x-\int_{\Omega} q_{\Omega} \operatorname{div} w \mathrm{~d} x=-D \tilde{J}_{0}\left(u_{\Omega}\right) w, \quad \forall w \in H_{0}^{1}(\Omega)^{3} .
$$


Corollary 5.1. Let $x_{0} \in \Omega$. Under the hypotheses of Theorem 5.1 and that $\alpha v_{\Omega}-\nu \Delta v_{\Omega}+\nabla q_{\Omega} \in L^{2}\left(D_{0}\right)^{3}$, we have

$$
\delta j\left(x_{0}\right)=A\left(u_{\Omega}\left(x_{0}\right)\right) \cdot v_{\Omega}\left(x_{0}\right)+\int_{D_{0}}\left(\alpha v_{\Omega}-\nu \Delta v_{\Omega}+\nabla q_{\Omega}\right)\left(R_{U}-S_{U}\right) \mathrm{d} x+\delta J\left(u_{0}\right) .
$$

Moreover in the particular case; $\omega$ is the unit ball $B(0,1), U(y), T(y)$ and $A\left(u_{\Omega}\left(x_{0}\right)\right)$ are given explicitly by

$$
\begin{aligned}
U(y) & =\pi \nu\left(6 G_{U}+\Delta G_{U}\right)(y) u_{\Omega}\left(x_{0}\right) \\
T(y) & =\frac{3 \nu}{2} u_{\Omega}\left(x_{0}\right), \quad \forall y \in \partial \omega \\
A\left(u_{\Omega}\left(x_{0}\right)\right) & =6 \pi \nu u_{\Omega}\left(x_{0}\right) .
\end{aligned}
$$

Proof. Thanks to Green's Formula together with (41) (with $S_{U}=R_{U}$ on $\Gamma_{R}$ ), (47) reads also

$$
\begin{aligned}
\delta j\left(x_{0}\right)= & \int_{\Gamma_{R}}\left[\left(\nu \nabla R_{U}-R_{P} I\right)-\left(\nu \nabla S_{U}-S_{P} I\right)\right] \cdot n v_{\Omega} \mathrm{d} \gamma(x)+\delta J\left(u_{0}\right) \\
= & \int_{\Gamma_{R}}\left(\nu \nabla v_{\Omega}-q_{\Omega} I\right) \cdot n S_{U} \mathrm{~d} \gamma(x)-\int_{\Gamma_{R}}\left(\nu \nabla S_{U}-S_{P} I\right) \cdot n v_{\Omega} \mathrm{d} \gamma(x) \\
& +\int_{D_{0}}\left(\alpha v_{\Omega}-\nu \Delta v_{\Omega}+\nabla q_{\Omega}\right) R_{U} \mathrm{~d} x-\alpha \int_{D_{0}} S_{U} v_{\Omega} \mathrm{d} x+\delta J\left(u_{0}\right) .
\end{aligned}
$$

Using a regularizing and localization technique, we derive

$$
\begin{aligned}
\int_{\Gamma_{R}}\left(\nu \nabla v_{\Omega}-q_{\Omega} I\right) \cdot n S_{U} \mathrm{~d} \gamma(x)-\int_{\Gamma_{R}}\left(\nu \nabla S_{U}-S_{P} I\right) . n & v_{\Omega} \mathrm{d} \gamma(x) \\
& =\int_{D_{0}}\left(\nu \Delta v_{\Omega}-\nabla q_{\Omega}\right) S_{U} \mathrm{~d} x-\left\langle\nu \Delta S_{U}-\nabla S_{P}, \varphi v_{\Omega}\right\rangle
\end{aligned}
$$

where $\varphi \in \mathcal{D}\left(D_{0}\right)$ satisfies $\varphi\left(x_{0}\right)=1$.

Finally, from (38) and (33) one can check that

$$
\begin{aligned}
\left\langle-\nu \Delta S_{U}+\nabla S_{P}, \varphi v_{\Omega}\right\rangle & =\left\langle-\nu \Delta\left(G_{U} A\left(u_{\Omega}\left(x_{0}\right)\right)\right)+\nabla\left(G_{P} \cdot A\left(u_{\Omega}\left(x_{0}\right)\right)\right), \varphi v_{\Omega}\right\rangle \\
& =\sum_{j} A_{j}\left(u_{\Omega}\left(x_{0}\right)\right)\left\langle\delta e_{j}, \varphi v_{\Omega}\right\rangle \\
& =A\left(u_{\Omega}\left(x_{0}\right)\right) \cdot v_{\Omega}\left(x_{0}\right),
\end{aligned}
$$

which proves (50).

For the case $\omega=B(0,1)$, one can derive the explicit expressions of the terms $U(y), T(y)$ and $A\left(u_{\Omega}\left(x_{0}\right)\right)$ from (32), (35) and

$$
\int_{\partial B(0,1)} G_{U}(y-x) \mathrm{d} \gamma(x)=\frac{2}{3 \nu} I, \quad \forall y \in \partial B(0,1) .
$$

For more details concerning the explicitly calculation of this terms, one may consult [24].

Now we discuss briefly the hypothesis (5) used in Theorem 5.1. It concerns the variation of the cost function $J_{\varepsilon}$. This question has been examined in [23] for the Dirichlet problem and in [24] for the Stokes problem. Here we limit ourselves to cost functions of the form

$$
\tilde{J}_{\varepsilon}(u)=\int_{\Omega_{\varepsilon}} g(x, u(x)) \mathrm{d} x, \quad u \in H^{1}\left(\Omega_{\varepsilon}\right)^{3}
$$

with $g$ is a given function defined on $\Omega \times \mathbb{R}^{3}$. 
The case where $g$ depends on the pressure $p$ is more complicated and we don't consider it in this paper. Next we suppose that $g$ satisfy the following hypotheses:

- for all $x \in \Omega$, the function $s \longmapsto g(x, s)$ is of class $\mathcal{C}^{1}$ on $\mathbb{R}^{3}$, its gradient being denoted by $\nabla_{s} g(x, s)$;

- for all $x \in \Omega$, the function $s \longmapsto \nabla_{s} g(x, s)$ is Lipschitz continuous and there exists a constant $M$ such that

$$
\left|\nabla_{s} g(x, t)-\nabla_{s} g(x, s)\right| \leq M|t-s|, \quad \forall(x, s, t) \in \Omega \times \mathbb{R}^{3} \times \mathbb{R}^{3}
$$

where $|t|$ denotes the usual norm on $\mathbb{R}^{n}$;

- the function $x \longmapsto \nabla_{s} g(x, 0)$ belongs to $L^{2}(\Omega)^{3}$ and $x \longmapsto g(x, 0)$ belongs to $L^{2}(\Omega)^{3 / 2}$.

These hypotheses imply that for all $(x, s) \in \Omega \times \mathbb{R}^{3}$

$$
\begin{aligned}
& |g(x, s)| \leq|g(x, 0)|+\left|\nabla_{s} g(x, 0) . s\right|+\frac{M}{2}|s|^{2} \\
& \left|\nabla_{s} g(x, s)\right| \leq\left|\nabla_{s} g(x, 0)\right|+M|s|,
\end{aligned}
$$

and the functions $x \longmapsto g(x, u(x))$ and $x \longmapsto\left|\nabla_{s} g(x, u(x))\right|^{2}$ are integrable on $\Omega$ for all $u \in L^{2}(\Omega)^{3}$.

A standard example of this functions is given by

$$
g(x, u)=\left|u(x)-\mathcal{U}_{g}(x)\right|^{2}
$$

The following result is taken from [24].

Proposition 5.1. Under the previous hypotheses and $f \in L^{2}(\Omega)^{3}$, we have

$$
\delta J\left(u_{0}\right)=\int_{D_{0}} \nabla_{s} g\left(x, u_{\Omega}\right)\left(R_{U}-S_{U}\right) \mathrm{d} x
$$

The adjoint state $\left(v_{\Omega}, q_{\Omega}\right) \in \mathcal{V}_{0} \times L_{0}^{2}(\Omega)$ is the solution to

$$
\alpha v_{\Omega}-\nu \Delta v_{\Omega}+\nabla q_{\Omega}=-\nabla_{s} g\left(x, u_{\Omega}\right)
$$

The function $j$ has the asymptotic expansion

$$
j(\varepsilon)=j(0)+\varepsilon A\left(u_{\Omega}\left(x_{0}\right)\right) v_{\Omega}\left(x_{0}\right)+o(\varepsilon) .
$$

If $\omega$ is the unit ball $B(1,0)$, then

$$
j(\varepsilon)=j(0)+6 \pi \nu \varepsilon u_{\Omega}\left(x_{0}\right) \cdot v_{\Omega}\left(x_{0}\right)+o(\varepsilon)
$$

\subsection{The two dimensional case}

In this paragraph, we intend to derive the asymptotic expansion of the function $j$ in the two dimensional case. The technique used is similar to that of the three dimensional case. We use the principal part of the Quasi-Stokes operator to derive the topological sensitivity expression. Next we briefly describe the transposition of the previous results to the two dimensional case. First, let us recall that $u_{\Omega}$ and the adjoint state $v_{\Omega}$ are respectively the solution to (8) and (48). 
Let $(U, P)$ be the solution to the Stokes exterior problem

$$
\left\{\begin{aligned}
-\nu \Delta U+\nabla P & =0 & & \text { in } \mathbb{R}^{2} \backslash \bar{\omega} \\
\operatorname{div} U & =0 & & \text { in } \mathbb{R}^{2} \backslash \bar{\omega} \\
U / \log \|y\| & =u_{\Omega}\left(x_{0}\right) & & \text { at infinity } \\
U & =0 & & \text { on } \partial \omega .
\end{aligned}\right.
$$

In $2 D$, the fundamental solution system to the Stokes equations is given by

$$
G_{U}(y)=\frac{1}{4 \pi \nu}\left(-(\log r) I+e_{r} e_{r}^{T}\right), \quad G_{P}(y)=\frac{y}{2 \pi r^{2}} .
$$

The functions $U$ and $P$ are written

$$
U(y)=u_{\Omega}\left(x_{0}\right) \log \|y\|+S_{U}(y)+L_{U}(y), \quad P(y)=S_{P}(y)+L_{P}(y)
$$

where at infinity $S_{U}(y)=O(1), L_{U}(y)=O(1 / r), S_{P}(y)=O(1 / r)$ and $L_{P}(y)=O\left(1 / r^{2}\right)$.

The associated interior problem consists in finding $\left(R_{U}, R_{P}\right)$ such that

$$
\left\{\begin{aligned}
\alpha R_{U}-\nu \Delta R_{U}+\nabla R_{P} & =\alpha S_{U} & & \text { in } D_{0} \\
\operatorname{div} R_{U} & =0 & & \text { in } D_{0} \\
R_{U} & =S_{U} & & \text { on } \Gamma_{R} .
\end{aligned}\right.
$$

In this case, a first order expansion of $\left.\left(u_{\varepsilon}^{f, \varphi}-u_{0}^{f, \varphi}\right)\right|_{D_{\varepsilon}}$ with $\varphi=\left.u_{\Omega}\right|_{\Gamma_{R}}$ is given by

$$
\left.\frac{-1}{\log \varepsilon}\left(u_{\Omega}\left(x_{0}\right) \log \frac{\|x\|}{R}+R_{U}-S_{U}\right)\right|_{D_{\varepsilon}} .
$$

Theorem 5.2. Under the same hypotheses of Theorem 5.1, the function $j$ has the following asymptotic expansion

$$
j(\varepsilon)=j(0)-\frac{1}{\log \varepsilon} \delta j\left(x_{0}\right)+o\left(\frac{1}{\log \varepsilon}\right)
$$

with

$$
\delta j\left(x_{0}\right)=\int_{\Gamma_{R}} \sigma\left(R_{U}-S_{U}\right) \cdot n v_{0} \mathrm{~d} \gamma(x)+\delta J\left(u_{0}\right)
$$

where $v_{0} \in \mathcal{V}_{R}$ is the solution to the adjoint equation

$$
a_{0}\left(w, v_{0}\right)=-D J_{0}\left(u_{0}\right) w, \quad \forall w \in \mathcal{V}_{R}
$$

And for a cost function of the form (56), we have:

Proposition 5.2. Let $\tilde{J}_{\varepsilon}$ a cost function of the form

$$
\tilde{J}_{\varepsilon}(u)=\int_{\Omega_{\varepsilon}} g(x, u(x)) \mathrm{d} x, \quad u \in H^{1}\left(\Omega_{\varepsilon}\right)^{2} .
$$

Under the same hypotheses of Proposition 5.1, the function $j$ has the following asymptotic expansion

$$
j(\varepsilon)=j(0)-\frac{4 \pi \nu u_{\Omega}\left(x_{0}\right) \cdot v_{\Omega}\left(x_{0}\right)}{\log \varepsilon}+o\left(\frac{1}{\log \varepsilon}\right) .
$$




\section{Numerical RESUlts}

Here, we limit ourselves to the two dimensional case. As application of the previous theoretical results, we present two examples. The first example concerns the identification of locations and orientations of several injectors in a water reserve. In the second example we treat the water eutrophication phenomena in a lake via dynamic aeration process.

In both cases, we deal with a cost function $J$ of the form

$$
J\left(u_{\Omega}\right)=\int_{\Omega_{m}}\left|u_{\Omega}-\mathcal{U}_{g}\right|^{2} \mathrm{~d} x
$$

with $\Omega_{m} \subset \Omega$ is the measurement domain and $\mathcal{U}_{g}$ is a given target flow.

Recall that we consider the Quasi-Stokes equations with a non homogeneous boundary condition on $\partial \omega_{\varepsilon}\left(u_{\Omega_{\varepsilon}}\right.$ $\left.=\mathcal{U}_{i n j}\right)($ see $(9))$. From (71) we deduce that

$$
\delta j(x)=\left(u_{\Omega}(x)-\mathcal{U}_{i n j}\right) \cdot v_{\Omega}(x), \quad \forall x \in \Omega
$$

where $u_{\Omega}$ and $v_{\Omega}$ are, respectively, solution to

$$
\begin{gathered}
\left\{\begin{aligned}
\alpha u_{\Omega}-\nu \Delta u_{\Omega}+\nabla p_{\Omega}=0 & \text { in } \Omega \\
\nabla \cdot u_{\Omega}=0 & \text { in } \Omega
\end{aligned}\right. \\
\left\{\begin{array}{cc}
\alpha v_{\Omega}-\nu \Delta v_{\Omega}+\nabla q_{\Omega}=-2\left(u_{\Omega}-\mathcal{U}_{g}\right) \chi_{\Omega_{m}} & \text { in } \Omega \\
\nabla \cdot v_{\Omega}=0 & \text { in } \Omega
\end{array}\right.
\end{gathered}
$$

where $\chi_{\Omega_{m}}$ is the characteristic function of the measurement domain.

Our implementation is based on the following optimization algorithm introduced by Céa et al. [6] and presented in the topological asymptotic context in [8].

\section{The algorithm:}

- initialization: choose $\Omega_{0}=\Omega_{d}$, and set $k=0$;

- repeat until target is reached:

- solve (73) and (74) in $\Omega_{k}$;

- compute the topological sensitivity $\delta j_{k}$;

- set $\Omega_{k+1}=\left\{x \in \Omega_{k}, \quad \delta j_{k}(x) \geq c_{k+1}\right\}$ where $c_{k+1}$ is chosen in such a way that the cost function decreases;

- $k \longleftarrow k+1$.

This algorithm can be seen as a descent method where the descent direction is determined by the topological sensitivity $\delta j_{k}$ and the step length is given by the volume variation.

We propose an adaptation of the previous algorithm to our context. We consider the set $\left\{x \in \Omega_{k} ; \delta j_{k}(x)<\right.$ $\left.c_{k+1}\right\}$. Each connected component of this set is a hole created by the algorithm. Our idea is to replace each hole by an injector located at the local minimum of $\delta j_{k}(x)$.

In the above algorithm, the systems (73) and (74) are discretized by a finite element method. The computation of the approximate solution is achieved by Uzawa algorithm.

\subsection{Test 1: identification of some injectors in a reserve water}

In this example, the computational domain $\Omega$ is a reserve water. Our purpose is to insert some injectors in $\Omega$ in order to reach a given target flow $\mathcal{U}_{g}$. Each injector $I n j_{k}$ is supposed as a small hole $\omega_{k}$ around $x_{k} \in \Omega$, 
having an injection velocity $U_{i n j}^{k}$. The velocity field $\mathcal{U}_{g}$ is chosen as the solution to $(73)$ in $\Omega_{l}=\Omega \backslash\left\{\cup_{k=1}^{l} \omega_{k}\right\}$, satisfying the following boundary conditions

$$
u= \begin{cases}U_{\text {wind }} & \text { on } \Gamma_{s} \\ 0 & \text { on } \Gamma_{w} \\ U_{\text {inj }}^{k} & \text { on } \partial \omega_{k}, \quad k=1, \ldots, l\end{cases}
$$

where $\Gamma_{s}$ is the free surface, $\Gamma_{w}$ is the wall and $U_{\text {wind }}$ is the velocity of wind.

Our aim here is to identify the locations and orientations of injectors from velocity measurement on the upper layer of $\Omega$. The magnitude of the velocity is known. The locations are given by the local minima of the topological sensitivity $\delta j$. From the $\delta j$ expression we deduce that the optimal orientations are given by the adjoint state $\left(\frac{v_{\Omega}}{\left\|v_{\Omega}\right\|}\right)$.

We consider here three cases, respectively one injector $(l=1)$, two injectors $(l=2)$ and three injectors $(l=3)$. For each case, we give a table summarizing the main parameters used to compute $\mathcal{U}_{g}$.

6.1.1. First case: one injector

\begin{tabular}{|c|c|c|}
\hline Injector & Location & Injection velocity \\
\hline Injector 1 & $x=0.1338773 E+01, y=0.2861623 E+00$ & $U_{x}=-0.8, U_{y}=-1.0$ \\
\hline
\end{tabular}

6.1.2. Second case: two injectors

\begin{tabular}{|c|c|c|}
\hline Injector & Location & Injection velocity \\
\hline Injector 1 & $x=0.1338773 E+01, y=0.2861623 E+00$ & $U_{x}=-0.8, U_{y}=-1.0$ \\
\hline Injector 2 & $x=0.6195151 E+00, y=0.2911333 E+00$ & $U_{x}=-1.0, U_{y}=1.3$ \\
\hline
\end{tabular}

6.1.3. Third case: three injectors

\begin{tabular}{|c|c|c|}
\hline Injector & Location & Injection velocity \\
\hline Injector 1 & $x=0.1338773 E+01, y=0.2861623 E+00$ & $U_{x}=-0.8, U_{y}=-1.0$ \\
\hline Injector 2 & $x=0.6195151 E+00, y=0.2911333 E+00$ & $U_{x}=-1.0, U_{y}=1.3$ \\
\hline Injector 3 & $x=0.8965202 E+00, y=0.5851877 E+00$ & $U_{x}=0.0, U_{y}=1.6$ \\
\hline
\end{tabular}

Using the previous algorithm, the numerical results that we present are obtained in one iteration.

In Figure 3, we present the initial flow, which is the same for the three considered cases. The results of this test are given by Figures 4-6. For each case, the injectors locations are given by the local minima of the topological sensitivity $\delta j$, see Figures $4 \mathrm{c}, 5 \mathrm{c}$ and $6 \mathrm{c}$. At each local minima, we introduce a pointwise Dirichlet condition (an injector inserted) and new resolution of (73) is performed. The new velocity obtained is shown in Figures $4 \mathrm{~d}, 5 \mathrm{~d}$ and $6 \mathrm{~d}$.

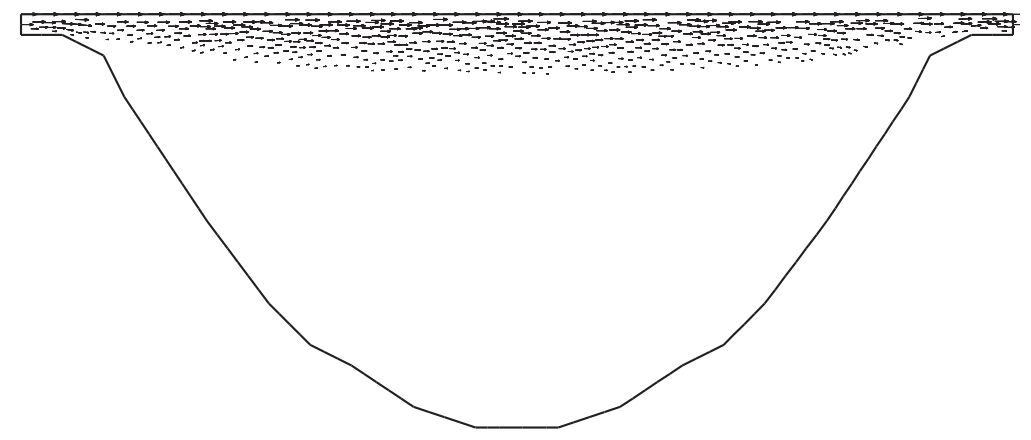

Figure 3 . The initial flow $u_{\Omega}$. 

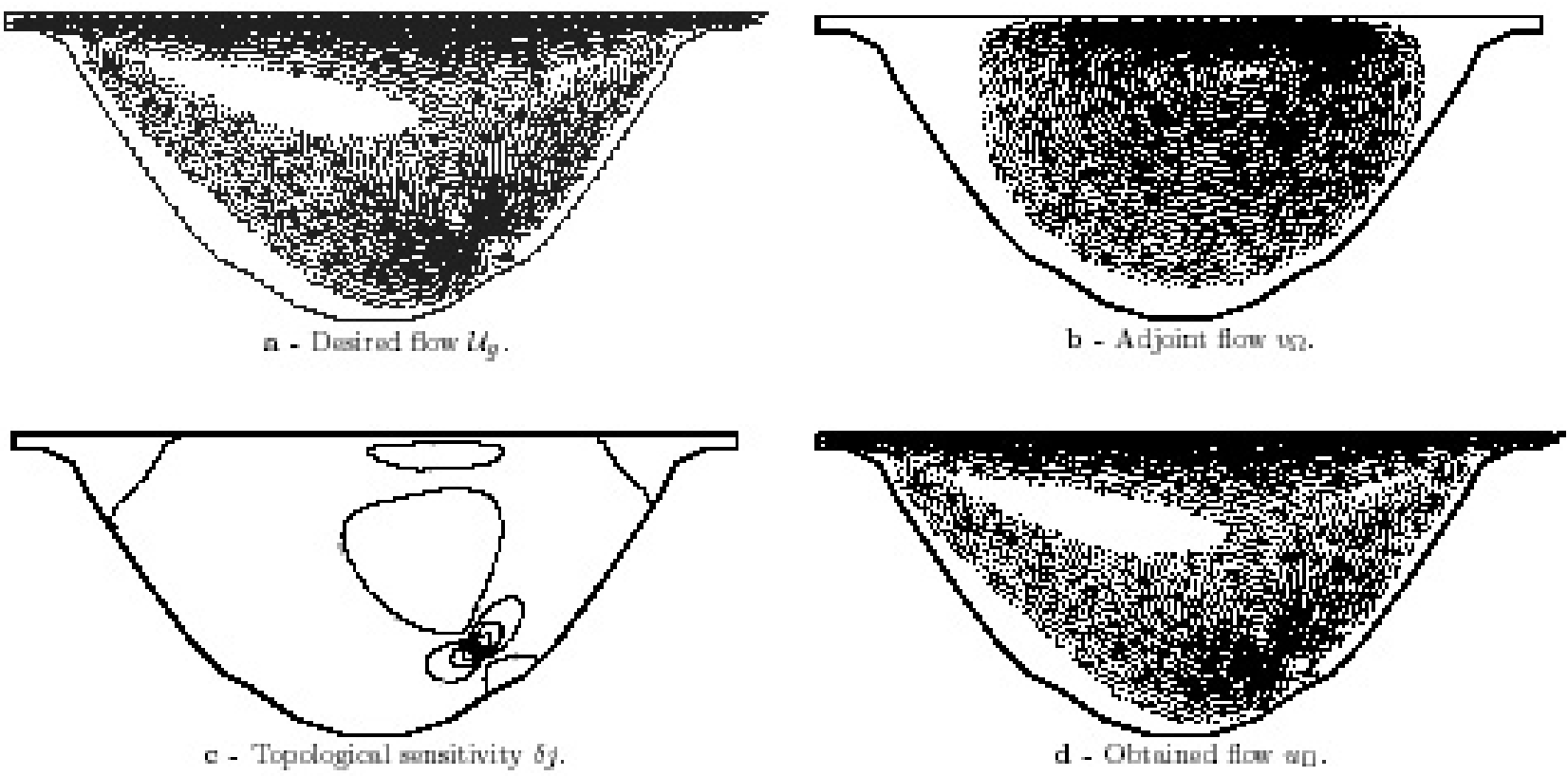

Figure 4. One injector case.
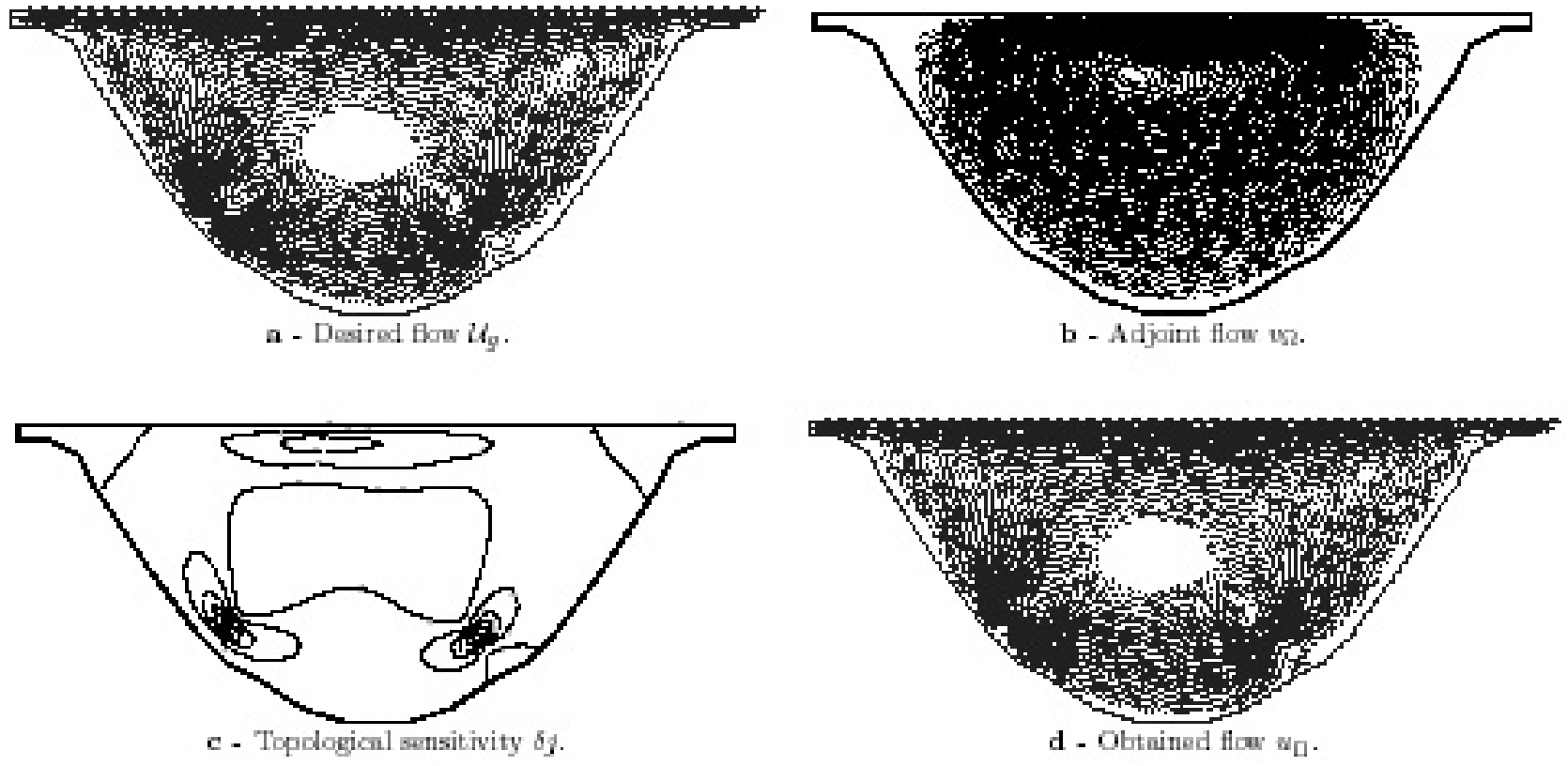

Figure 5. Two injectors case.

\subsection{Test 2: dynamic aeration process in an eutrophized lake}

Here, the computational domain $\Omega$ is an eutrophized lake. In this example, we treat the water eutrophication phenomena by dynamic aeration process. It consists in inserting some injector holes $\omega_{k}$ at the lower layer of 

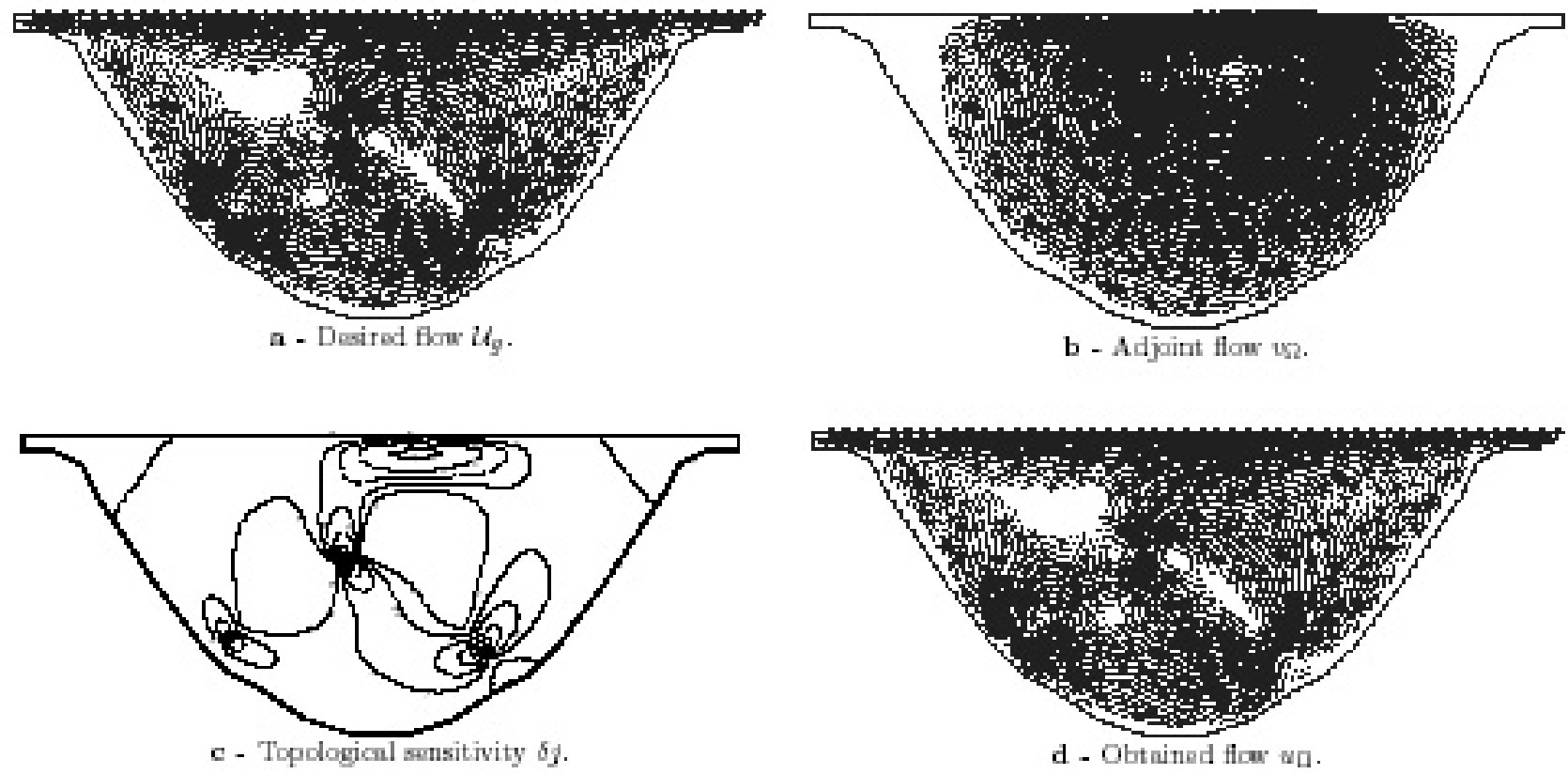

FiguRE 6. Three injectors case.

the lake in order to create a motion mixing the bottom water with the well oxygenated water from the top. We suppose that a "good" lake oxygenation can be described by a target velocity $\mathcal{U}_{g}$. Our aim in this test, is to determine the optimal location in $\Omega$ of some injector holes $\omega_{k}$ in order to minimize the function $\int_{\Omega_{l}}\left|u_{\Omega}-\mathcal{U}_{g}\right|^{2} \mathrm{~d} x$, with $\Omega_{l}=\Omega \backslash\left\{\cup_{k=1}^{l} \omega_{k}\right\}$.

After only sixth iterations, we obtain a velocity (see Fig. 7) approaching the objective flow $\mathcal{U}_{g}$. We present for each iteration $l=1,6$ the injector location and the obtained flow $u_{\Omega_{l}}$. Then, Figure 7 shows the initial, desired and the obtained flow. In order to have more idea of what is happening, we represent on Figure 8 several intermediate injectors locations and velocity obtained during the optimization process.

This work can be considered as a preliminary step to study the transient Navier-Stokes problem.

\section{VARIATIONS OF THE BILINEAR AND LINEAR FORM}

We now turn to the proof of the main results given by Theorem 5.1. We will use the result given in [15, 29], which is recalled in Section 2. More precisely, we will prove in Sections 7.3 and 7.4 that there exist a bilinear form $\delta a \in \mathcal{L}_{2}\left(\mathcal{V}_{R}\right)$ and a linear form $\delta l \in \mathcal{L}\left(\mathcal{V}_{R}\right)$ such that

$$
\begin{gathered}
\left\|a_{\varepsilon}-a_{0}-\varepsilon \delta a\right\|_{\mathcal{L}_{2}\left(\mathcal{V}_{R}\right)}=O\left(\varepsilon^{3 / 2}\right), \\
\left\|l_{\varepsilon}-l_{0}-\varepsilon \delta l\right\|_{\mathcal{L}\left(\mathcal{V}_{R}\right)}=O\left(\varepsilon^{3 / 2}\right) .
\end{gathered}
$$

First we need some definitions and preliminary lemmas. 


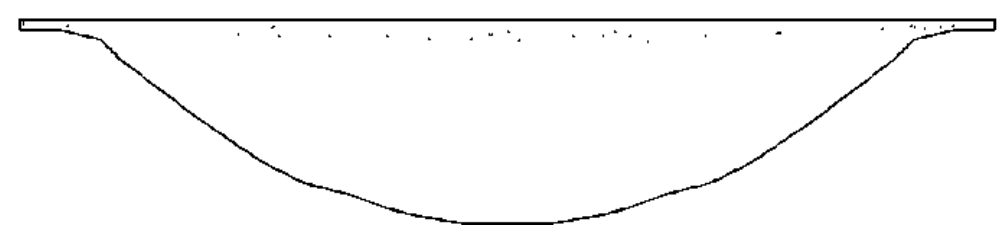

a - The initial flow $u_{\Omega}$.

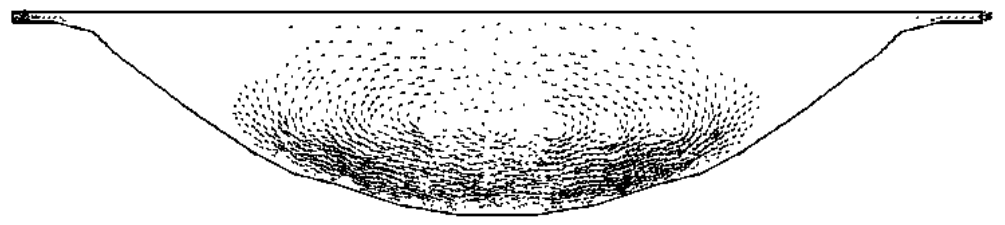

b - The desired flow $\mathcal{U}_{g}$.

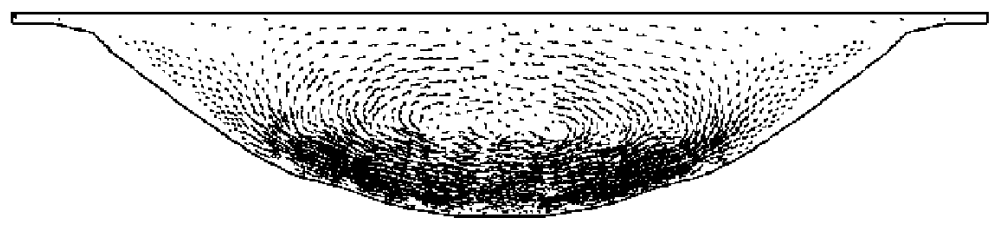

c - The obtained flow $u_{\Omega}$ (after sixth iterations).

FigurE 7. The initial, desired and obtained flow.

\subsection{Definitions}

Let $\mathcal{O}$ be a bounded open domain of $\mathbb{R}^{3}$ and $\partial \mathcal{O}$ its boundary, assumed polygonal and simply connected.

- $H^{m}(\mathcal{O})^{3}$ stands for the Hilbert Sobolev space of order $m$, where $m$ is a positive integer. It is provided with the norm

$$
\|u\|_{m, \mathcal{O}}^{2}=\sum_{k=0}^{m}|u|_{k, \mathcal{O}}^{2}
$$

where the semi-norms $|\cdot|_{k, \mathcal{O}}$ are defined

$$
|u|_{k, \mathcal{O}}^{2}=\sum_{|\alpha|=k} \int_{\mathcal{O}}\left|\partial_{\alpha} u\right|^{2} \mathrm{~d} x
$$

- The usual space of traces (of $H^{1}(\mathcal{O})$ elements) on the boundary of $\mathcal{O}$ is denoted $H^{1 / 2}(\partial \mathcal{O})$, and its norm is denoted by $\|\cdot\|_{1 / 2, \partial \mathcal{O}}$. The subspace

$$
H_{V}^{1 / 2}(\partial \mathcal{O})^{3}=\left\{\varphi \in H^{1 / 2}(\partial \mathcal{O})^{3} ; \quad \int_{\partial \mathcal{O}} \varphi \cdot n \mathrm{~d} \gamma(x)=0\right\}
$$

is equipped with the norm induced by $H^{1 / 2}(\partial \mathcal{O})^{3}$ and $H_{V}^{-1 / 2}(\partial \mathcal{O})^{3}$ denotes its dual space. 

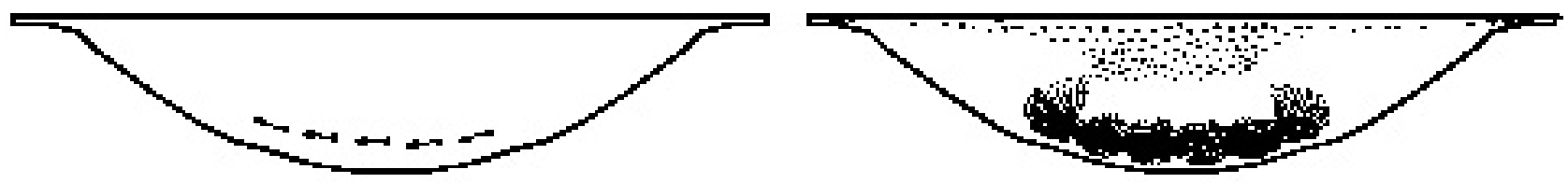

a - First iteration,
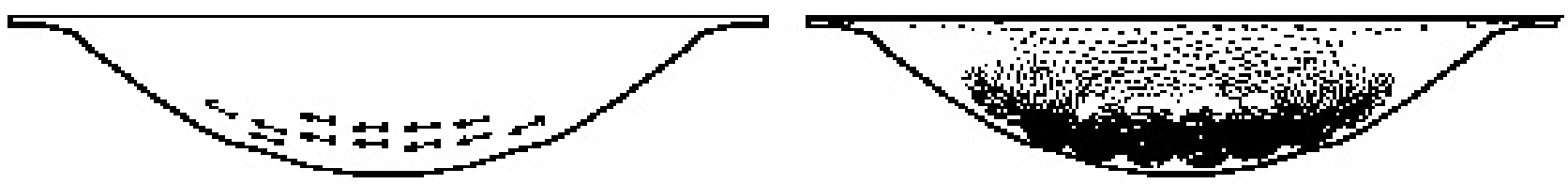

b - Sercod iterntion.
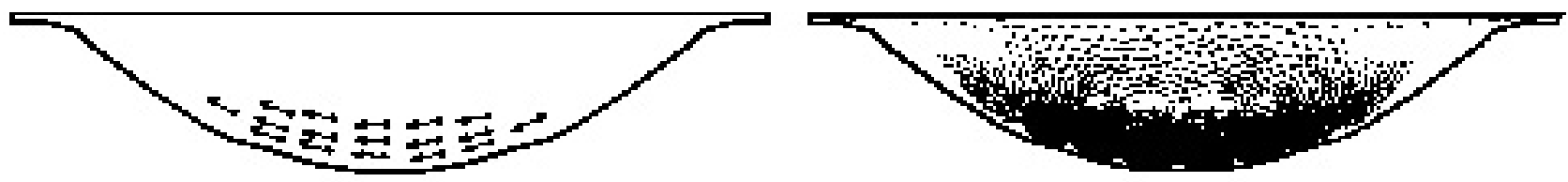

c - Third iteration.
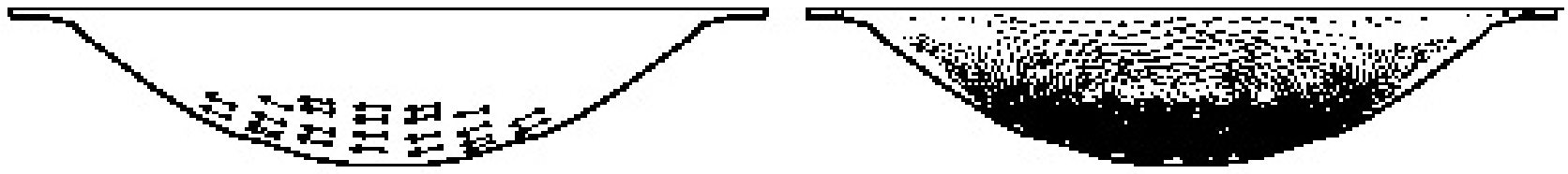

d - Fourth iteration.
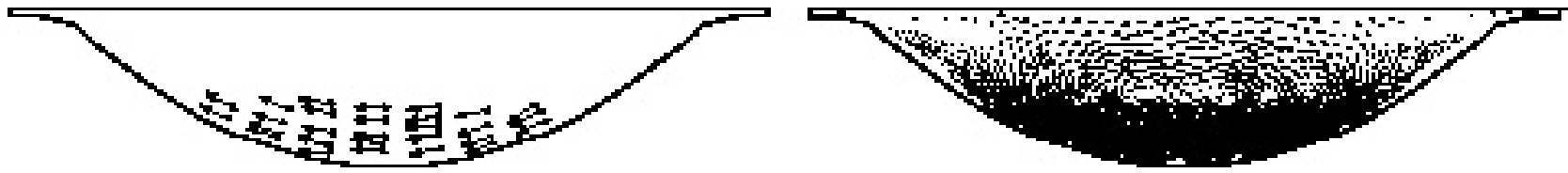

e. Fifth iteraticn.
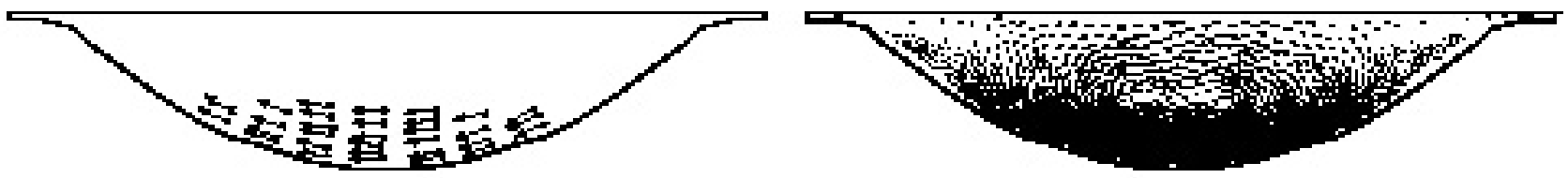

f - Sixth iteration.

FigURE 8. Injectors locations and velocity obtained during optimization process.

- Let $\varepsilon>0$; for a given function $u$ defined on $\mathcal{O}$, we define the function $\tilde{u}$ on $\tilde{\mathcal{O}}:=\mathcal{O} / \varepsilon$ by

$$
\tilde{u}(y)=u(x), \quad y=x / \varepsilon .
$$


Using that $D u(x)=D \tilde{u}(y) / \varepsilon$ and the definition (79), we obtain

$$
|u|_{1, \mathcal{O}}^{2}=\int_{\mathcal{O}}|D u(x)|^{2} \mathrm{~d} x=1 / \varepsilon \int_{\tilde{\mathcal{O}}}|D \tilde{u}(y)|^{2} \mathrm{~d} y,
$$

hence

Similarly, we have

$$
|u|_{1, \mathcal{O}}=\varepsilon^{1 / 2}|\tilde{u}|_{1, \tilde{\mathcal{O}}}
$$

$$
\|u\|_{0, \mathcal{O}}=\varepsilon^{3 / 2}\|\tilde{u}\|_{0, \tilde{\mathcal{O}}} .
$$

\subsection{Preliminary lemmas}

The aim of this section is to give some technical results which will be used in Sections 7.3 and 7.4. Let us begin by recalling some estimates describing the behavior of the two parts $S_{U}$ and $L_{U}$ of the fundamental solution $U$, see (37).

Lemma $7.1[15]$. For $\phi \in H_{V}^{1 / 2}(\partial \omega)^{3}$; let $U, P$ be the solution to the Stokes exterior problem

$$
\left\{\begin{aligned}
-\nu \Delta U+\nabla Q=0 & \text { in } \mathbb{R}^{3} \backslash \bar{\omega} \\
\operatorname{div} U=0 & \text { in } \mathbb{R}^{3} \backslash \bar{\omega} \\
U=0 & \text { at infinity } \\
U=\phi & \text { on } \partial \omega .
\end{aligned}\right.
$$

The function $U$ is splited into

$$
\begin{aligned}
U(y)=S_{U}(y)+L_{U}(y) \quad \forall y \in \mathbb{R}^{3} \backslash \bar{\omega} \\
\text { with } \quad S_{U}(y)=G_{U}(y) \int_{\partial \omega} T(x) \mathrm{d} \gamma(x)
\end{aligned}
$$

where $G_{U}(y)$ is defined in (32) and $T \in H_{V}^{-1 / 2}(\partial \omega)^{3}$ is the unique solution [11] to

$$
\int_{\partial \omega} G_{U}(y-x) T(x) \mathrm{d} \gamma(x)=\phi(y), \quad \forall y \in \partial \omega .
$$

There exists a constant $c>0$, independent of $\phi$ and $\varepsilon$, such that

$$
\begin{aligned}
\left\|S_{U}\right\|_{0, C(R /(2 \varepsilon), R / \varepsilon)} & \leq c \varepsilon^{-1 / 2}\|\phi\|_{1 / 2, \partial \omega} \\
\left\|S_{U}\right\|_{0, D_{\varepsilon} / \varepsilon} & \leq c \varepsilon^{-1 / 2}\|\phi\|_{1 / 2, \partial \omega} \\
\left|S_{U}\right|_{1, C(R /(2 \varepsilon), R / \varepsilon)} & \leq c \varepsilon^{1 / 2}\|\phi\|_{1 / 2, \partial \omega} \\
\left|S_{U}\right|_{1, D_{\varepsilon} / \varepsilon} & \leq c\|\phi\|_{1 / 2, \partial \omega} \\
\left\|L_{U}\right\|_{0, C(R /(2 \varepsilon), R / \varepsilon)} & \leq c \varepsilon^{1 / 2}\|\phi\|_{1 / 2, \partial \omega} \\
\left|L_{U}\right|_{1, C(R /(2 \varepsilon), R / \varepsilon)} & \leq c \varepsilon^{3 / 2}\|\phi\|_{1 / 2, \partial \omega} \\
\left\|L_{U}\right\|_{1, D_{\varepsilon} / \varepsilon} & \leq c\|\phi\|_{1 / 2, \partial \omega} .
\end{aligned}
$$

Lemma 7.2. For a given $\varepsilon>0$ and $\varphi \in H_{V}^{1 / 2}\left(\Gamma_{R}\right)^{3}$, let $v_{\varepsilon}, q_{\varepsilon}$ be the solution to the problem

$$
\left\{\begin{aligned}
\alpha v_{\varepsilon}-\nu \Delta v_{\varepsilon}+\nabla q_{\varepsilon}=0 & & \text { in } D_{\varepsilon} \\
\operatorname{div} v_{\varepsilon}=0 & & \text { in } D_{\varepsilon} \\
v_{\varepsilon}=\varphi & & \text { on } \Gamma_{R} \\
v_{\varepsilon}=0 & & \text { on } \partial \omega_{\varepsilon} .
\end{aligned}\right.
$$


Then, there exists two constants $c>0$ (independent of $\varphi$ and $\varepsilon$ ) and $\varepsilon_{1}>0$ such that for all $0<\varepsilon<\varepsilon_{1}$, we have

$$
\left\|v_{\varepsilon}\right\|_{1, D_{\varepsilon}} \leq c\|\varphi\|_{1 / 2, \Gamma_{R}} .
$$

Proof. For all $\varepsilon>0$, problem (86) is well posed and the solution $v_{\varepsilon} \in H^{1}\left(D_{\varepsilon}\right)^{3}$. Particularly for a given $\varepsilon_{0}>0$, $v_{\varepsilon_{0}} \in H^{1}\left(D_{\varepsilon_{0}}\right)^{3}$ and there exists a constant $c>0$ such that

$$
\left\|v_{\varepsilon_{0}}\right\|_{1, D_{\varepsilon_{0}}} \leq c\|\varphi\|_{1 / 2, \Gamma_{R}} .
$$

Let $\varepsilon_{1}<\varepsilon_{0}$ be such that $D_{\varepsilon_{0}} \subset D_{\varepsilon}$ for all $\varepsilon<\varepsilon_{1}$, and let $\tilde{v}_{\varepsilon_{0}}$ be the extension of $v_{\varepsilon_{0}}$ to $D_{\varepsilon}$ by 0 . The function $v_{\varepsilon}$ is solution to the Quasi-Stokes problem (86), it can be shown also as a solution to the following minimization problem

$$
\min _{v \in \mathcal{U}}\left\{\alpha\|v\|_{0, D_{\varepsilon}}+\nu|v|_{1, D_{\varepsilon}}\right\}
$$

where $\mathcal{U}=\left\{v \in H^{1}\left(D_{\varepsilon}\right)^{3} ; v=\varphi\right.$ on $\Gamma_{R}$, $\operatorname{div} v=0$ in $D_{\varepsilon}$ and $v=0$ on $\left.\partial \omega_{\varepsilon}\right\}$. Hence, for all $\varepsilon<\varepsilon_{1}$ we have

$$
\left\|v_{\varepsilon}\right\|_{1, D_{\varepsilon}} \leq\left\|\tilde{v}_{\varepsilon_{0}}\right\|_{1, D_{\varepsilon}}=\left\|v_{\varepsilon_{0}}\right\|_{1, D_{\varepsilon_{0}}} \leq c\|\varphi\|_{1 / 2, \Gamma_{R}} .
$$

Lemma 7.3. For $\varepsilon>0$ and $\psi \in H^{1}\left(D_{0}\right)^{3}$ such that, $\operatorname{div} \psi=0$, let $u_{\varepsilon}, p_{\varepsilon}$ be the solution to the Quasi-Stokes problem

$$
\left\{\begin{aligned}
\alpha u_{\varepsilon}-\nu \Delta u_{\varepsilon}+\nabla p_{\varepsilon}=0 & \text { in } D_{\varepsilon} \\
\operatorname{div} u_{\varepsilon}=0 & \text { in } D_{\varepsilon} \\
u_{\varepsilon}=0 & \text { on } \Gamma_{R} \\
u_{\varepsilon}=\psi & \text { on } \partial \omega_{\varepsilon} .
\end{aligned}\right.
$$

Then, there exists two constants $c>0$ (independent of $\psi$ and $\varepsilon$ ) and $\varepsilon_{1}>0$ such that for all $0<\varepsilon<\varepsilon_{1}$

$$
\begin{aligned}
\left|u_{\varepsilon}\right|_{1, C(R / 2, R)} & \leq c \varepsilon\|\psi(\varepsilon y)\|_{1 / 2, \partial \omega} \\
\left\|u_{\varepsilon}\right\|_{0, D_{\varepsilon}} & \leq c \varepsilon\|\psi(\varepsilon y)\|_{1 / 2, \partial \omega} \\
\left|u_{\varepsilon}\right|_{1, D_{\varepsilon}} & \leq c \varepsilon^{1 / 2}\|\psi(\varepsilon y)\|_{1 / 2, \partial \omega} .
\end{aligned}
$$

Proof. First, we denote by $\left(V_{\varepsilon}, Q_{\varepsilon}\right)$ the solution to the exterior problem (83) obtained using $\phi(y)=\psi(\varepsilon y)$ on the boundary $\partial \omega$.

Posing $v_{\varepsilon}=V_{\varepsilon}(x / \varepsilon)$, then the function $w_{\varepsilon}=v_{\varepsilon}-u_{\varepsilon}$ itself is the solution to

$$
\left\{\begin{aligned}
\alpha w_{\varepsilon}-\nu \Delta w_{\varepsilon}+\nabla s_{\varepsilon} & =\alpha v_{\varepsilon} & & \text { in } D_{\varepsilon} \\
\operatorname{div} w_{\varepsilon} & =0 & & \text { in } D_{\varepsilon} \\
w_{\varepsilon} & =v_{\varepsilon} & & \text { on } \Gamma_{R} \\
w_{\varepsilon} & =0 & & \text { on } \partial \omega_{\varepsilon} .
\end{aligned}\right.
$$

Thanks to Lemma 7.2 and elliptic regularity it can be shown that there exists $c>0$ and $\varepsilon_{1}>0$ such that for all $0<\varepsilon<\varepsilon_{1}$

$$
\left\|w_{\varepsilon}\right\|_{1, D_{\varepsilon}} \leq c\left(\left\|v_{\varepsilon}\right\|_{1 / 2, \Gamma_{R}}+\alpha\left\|v_{\varepsilon}\right\|_{0, D_{\varepsilon}}\right) .
$$

In the other hand, we have

$$
\left\|v_{\varepsilon}\right\|_{1 / 2, \Gamma_{R}} \leq\left\|v_{\varepsilon}\right\|_{1, C(R / 2, R)} \leq\left\|v_{\varepsilon}\right\|_{0, C(R / 2, R)}+\left|v_{\varepsilon}\right|_{1, C(R / 2, R)} .
$$


From (81), (82) and Lemma 7.1, we obtain

$$
\begin{aligned}
& \left\|v_{\varepsilon}\right\|_{0, C(R / 2, R)}=\varepsilon^{3 / 2}\left\|V_{\varepsilon}\right\|_{0, C(R / 2 \varepsilon, R / \varepsilon)} \leq c \varepsilon\|\psi(\varepsilon y)\|_{1 / 2, \partial \omega}, \\
& \left|v_{\varepsilon}\right|_{1, C(R / 2, R)}=\varepsilon^{1 / 2}\left|V_{\varepsilon}\right|_{1, C(R / 2 \varepsilon, R / \varepsilon)} \leq c \varepsilon\|\psi(\varepsilon y)\|_{1 / 2, \partial \omega} .
\end{aligned}
$$

Hence,

Similarly we get

$$
\left\|v_{\varepsilon}\right\|_{1 / 2, \Gamma_{R}} \leq c \varepsilon\|\psi(\varepsilon y)\|_{1 / 2, \partial \omega} .
$$

$$
\begin{aligned}
& \left\|v_{\varepsilon}\right\|_{0, D_{\varepsilon}}=\varepsilon^{3 / 2}\left\|V_{\varepsilon}\right\|_{0, D_{\varepsilon} / \varepsilon} \leq c \varepsilon\|\psi(\varepsilon y)\|_{1 / 2, \partial \omega}, \\
& \left|v_{\varepsilon}\right|_{1, D_{\varepsilon}}=\varepsilon^{1 / 2}\left|V_{\varepsilon}\right|_{1, D_{\varepsilon} / \varepsilon} \leq c \varepsilon^{1 / 2}\|\psi(\varepsilon y)\|_{1 / 2, \partial \omega} .
\end{aligned}
$$

Relation (92), (93) and (94) implies that

$$
\left\|w_{\varepsilon}\right\|_{1, D_{\varepsilon}} \leq c \varepsilon\|\psi(\varepsilon y)\|_{1 / 2, \partial \omega} .
$$

The desired results follows from the following inequalities

$$
\begin{aligned}
\left|u_{\varepsilon}\right|_{1, C(R / 2, R)}=\left|v_{\varepsilon}-w_{\varepsilon}\right|_{1, C(R / 2, R)} & \leq\left|v_{\varepsilon}\right|_{1, C(R / 2, R)}+\left|w_{\varepsilon}\right|_{1, C(R / 2, R)} \\
& \leq c \varepsilon\|\psi(\varepsilon y)\|_{1 / 2, \partial \omega} \\
\left\|u_{\varepsilon}\right\|_{0, D_{\varepsilon}} \leq\left\|v_{\varepsilon}\right\|_{0, D_{\varepsilon}}+\left\|w_{\varepsilon}\right\|_{0, D_{\varepsilon}} & \leq c \varepsilon\|\psi(\varepsilon y)\|_{1 / 2, \partial \omega} \\
\left|u_{\varepsilon}\right|_{1, D_{\varepsilon}} \leq\left|v_{\varepsilon}\right|_{1, D_{\varepsilon}}+\left|w_{\varepsilon}\right|_{1, D_{\varepsilon}} & \leq c \varepsilon^{1 / 2}\|\psi(\varepsilon y)\|_{1 / 2, \partial \omega}+c \varepsilon\|\psi(\varepsilon y)\|_{1 / 2, \partial \omega} \\
& \leq c \varepsilon^{1 / 2}\|\psi(\varepsilon y)\|_{1 / 2, \partial \omega} .
\end{aligned}
$$

The following lemma summarize the results shown in Lemmas 7.2 and 7.3.

Lemma 7.4. For a given $\varepsilon>0, h_{\varepsilon} \in L^{2}\left(D_{\varepsilon}\right)^{3}, \varphi \in H_{V}^{1 / 2}(\Gamma)^{3}$ and $\psi \in H^{1}\left(D_{0}\right)^{3}$ such that, div $\psi=0$, let $v_{\varepsilon}, q_{\varepsilon}$ be the solution to the Quasi-Stokes problem

$$
\left\{\begin{aligned}
\alpha v_{\varepsilon}-\nu \Delta v_{\varepsilon}+\nabla q_{\varepsilon} & =h_{\varepsilon} & & \text { in } D_{\varepsilon} \\
\operatorname{div} v_{\varepsilon} & =0 & & \text { in } D_{\varepsilon} \\
v_{\varepsilon} & =\varphi & & \text { on } \Gamma_{R} \\
v_{\varepsilon} & =\psi & & \text { on } \partial \omega_{\varepsilon}
\end{aligned}\right.
$$

Then, there exists a constant $c>0$ (independent of $\varphi, \psi$ and $\varepsilon$ ), and $\varepsilon_{1}>0$ such that for all $0<\varepsilon<\varepsilon_{1}$

$$
\begin{aligned}
\left|v_{\varepsilon}\right|_{1, C(R / 2, R)} & \leq c\left(\|\varphi\|_{1 / 2, \Gamma_{R}}+\varepsilon\|\psi(\varepsilon y)\|_{1 / 2, \partial \omega}+\left\|h_{\varepsilon}\right\|_{0, D_{\varepsilon}}\right) \\
\left\|v_{\varepsilon}\right\|_{0, D_{\varepsilon}} & \leq c\left(\|\varphi\|_{1 / 2, \Gamma_{R}}+\varepsilon\|\psi(\varepsilon y)\|_{1 / 2, \partial \omega}+\left\|h_{\varepsilon}\right\|_{0, D_{\varepsilon}}\right) \\
\left|v_{\varepsilon}\right|_{1, D_{\varepsilon}} & \leq c\left(\|\varphi\|_{1 / 2, \Gamma_{R}}+\varepsilon^{1 / 2}\|\psi(\varepsilon y)\|_{1 / 2, \partial \omega}+\left\|h_{\varepsilon}\right\|_{0, D_{\varepsilon}}\right) .
\end{aligned}
$$

Proof. The proof follows easily by combining Lemmas 7.2 and 7.3 and using the linearity of the Quasi-Stokes operator.

\subsection{Variation of the bilinear form}

Let us now compute the variation of the bilinear form $a_{\varepsilon}$ with respect to the creation of a small hole $\omega_{\varepsilon}$ in the domain $\Omega$. Then, according to (20), we have

$$
a_{\varepsilon}(u, v)-a_{0}(u, v)=\int_{\Gamma_{R}}\left(T_{\varepsilon}-T_{0}\right) u . v \mathrm{~d} \gamma(x) .
$$


So, we first need to compute the operator $T_{\varepsilon}$ variation. To this aim, we introduce some notations.

For $\varphi \in H_{V}^{1 / 2}\left(\Gamma_{R}\right)$, recall that $u_{\varepsilon}^{0, \varphi}$ is the solution to $(13)$ or $(14)$ if $\varepsilon=0$. We denote by $\left(U^{0, \varphi}, P^{0, \varphi}\right)$ the solution to the exterior problem $(83)$ with $\phi=u_{0}^{0, \varphi}\left(x_{0}\right)$ on the boundary $\partial \omega$.

As in $(37),\left(U^{0, \varphi}, P^{0, \varphi}\right)$ can be decomposed into two parts:

$$
U^{0, \varphi}=S_{U}^{0, \varphi}+L_{U}^{0, \varphi}, \quad P^{0, \varphi}=S_{P}^{0, \varphi}+L_{P}^{0, \varphi}
$$

with $S_{U}^{0, \varphi}=G_{U}(y) A\left(u_{0}^{0, \varphi}\left(x_{0}\right)\right), S_{P}^{0, \varphi}=G_{P}(y) A\left(u_{0}^{0, \varphi}\left(x_{0}\right)\right)$ are the dominant parts respectively of $U^{0, \varphi}$ and $P^{0, \varphi}$. Now, let $R_{U}^{0, \varphi}$ be the solution to the associated interior problem

$$
\left\{\begin{aligned}
\alpha R_{U}^{0, \varphi}-\nu \Delta R_{U}^{0, \varphi}+\nabla R_{P}^{0, \varphi} & =\alpha S_{U}^{0, \varphi} & & \text { in } D_{0} \\
\operatorname{div} R_{U}^{0, \varphi} & =0 & & \text { in } D_{0} \\
R_{U}^{0, \varphi} & =S_{U}^{0, \varphi} & & \text { on } \Gamma_{R} .
\end{aligned}\right.
$$

Then, the linear operator $\delta T$ (independent of $\varepsilon$ ) is defined as follows:

$$
\begin{aligned}
\delta T: H_{V}^{1 / 2}\left(\Gamma_{R}\right)^{3} & \longrightarrow H_{V}^{-1 / 2}\left(\Gamma_{R}\right)^{3} \\
\varphi & \rightarrow \delta T \varphi=\sigma\left(R_{U}^{0, \varphi}-S_{U}^{0, \varphi}\right) . n .
\end{aligned}
$$

Proposition 7.1. The operator $T_{\varepsilon}$ has the following asymptotic expansion

$$
\left\|T_{\varepsilon}-T_{0}-\varepsilon \delta T\right\|_{\mathcal{L}\left(H_{V}^{1 / 2}\left(\Gamma_{R}\right)^{3} ; H_{V}^{-1 / 2}\left(\Gamma_{R}\right)^{3}\right)}=O\left(\varepsilon^{3 / 2}\right) .
$$

Proof. As we have shown in Section 5 (see (37), (38), (40)); for $y=x / \varepsilon$, we have

$$
S_{U}^{0, \varphi}(x / \varepsilon)=\varepsilon S_{U}^{0, \varphi}(x), L_{U}^{0, \varphi}(y)=O\left(1 /\|y\|^{2}\right), S_{P}^{0, \varphi}(x / \varepsilon)=\varepsilon^{2} S_{P}^{0, \varphi}(x), \text { and } L_{P}^{0, \varphi}(y)=O\left(1 /\|y\|^{3}\right) .
$$

Next, for simplicity, we may drop the subscripts $(.)^{0, \varphi}$. Let

$$
\psi_{\varepsilon}(x)=\left(T_{\varepsilon}-T_{0}-\varepsilon \delta T\right) \varphi(x) .
$$

We have

$$
\begin{aligned}
\psi_{\varepsilon} & =\sigma\left(u_{\varepsilon}-u_{0}\right) \cdot n-\varepsilon \sigma\left(R_{U}-S_{U}\right) . n \\
& =\left(\nu \nabla u_{\varepsilon}-p_{\varepsilon} I\right) \cdot n-\left(\nu \nabla u_{0}-p_{0} I\right) . n-\varepsilon\left[\left(\nu \nabla R_{U}-R_{P} I\right) . n-\left(\nu \nabla S_{U}-S_{P} I\right) . n\right] .
\end{aligned}
$$

Posing

Then, $\psi_{\varepsilon}$ is written as

$$
\begin{aligned}
& w_{\varepsilon}=u_{\varepsilon}-u_{0}+U(x / \varepsilon)-\varepsilon R_{U} \\
& s_{\varepsilon}=p_{\varepsilon}-p_{0}+1 / \varepsilon P(x / \varepsilon)-\varepsilon R_{P}(x) .
\end{aligned}
$$

$$
\psi_{\varepsilon}=\nu \nabla\left(w_{\varepsilon}(x)-L_{U}(x / \varepsilon)\right) . n-\left(s_{\varepsilon}(x)-1 / \varepsilon L_{P}(x / \varepsilon)\right) I . n=\sigma\left(w_{\varepsilon}(x)-L_{U}(x / \varepsilon)\right) . n .
$$

The functions $w_{\varepsilon}, s_{\varepsilon}$ are solution to

$$
\left\{\begin{aligned}
\alpha w_{\varepsilon}-\nu \Delta w_{\varepsilon}+\nabla s_{\varepsilon} & =\alpha L_{U}(x / \varepsilon) & & \text { in } D_{\varepsilon} \\
\operatorname{div} w_{\varepsilon} & =0 & & \text { in } D_{\varepsilon} \\
w_{\varepsilon} & =U(x / \varepsilon)-\varepsilon R_{U}(x) & & \text { on } \Gamma_{R} \\
w_{\varepsilon} & =-u_{0}(x)+u_{0}\left(x_{0}\right)-\varepsilon R_{U}(x) & & \text { on } \partial \omega_{\varepsilon} .
\end{aligned}\right.
$$


In order to use Lemma 7.4, we need to estimate the right hand side terms. For the boundary terms, we will proceed in the same way as in [24] for the Stokes problem.

- On $\Gamma_{R}$, due to $R_{U}(x)=S_{U}(x)$, we have

$$
U(x / \varepsilon)-\varepsilon R_{U}(x)=L_{U}(x / \varepsilon)
$$

and from the definitions of the functions $U$ and $R_{U}$, we get

$$
\int_{\Gamma_{R}}\left(U(x / \varepsilon)-\varepsilon R_{U}(x)\right) \cdot n \mathrm{~d} \gamma(x)=0 .
$$

Using (81), (82) and Lemma 7.1, we check that

$$
\begin{aligned}
\left\|U(x / \varepsilon)-\varepsilon R_{U}(x)\right\|_{1 / 2, \Gamma_{R}} & =\left\|L_{U}(x / \varepsilon)\right\|_{1 / 2, \Gamma_{R}} \\
& \leq c\left\|L_{U}(x / \varepsilon)\right\|_{1, C(R / 2, R)} \\
& \leq c\left(\left\|L_{U}(x / \varepsilon)\right\|_{0, C(R / 2, R)}+\left|L_{U}(x / \varepsilon)\right|_{1, C(R / 2, R)}\right) \\
& \leq c\left(\varepsilon^{3 / 2}\left\|L_{U}(y)\right\|_{0, C(R / 2 \varepsilon, R / \varepsilon)}+\varepsilon^{1 / 2}\left|L_{U}(y)\right|_{1, C(R / 2 \varepsilon, R / \varepsilon)}\right) \\
& \leq c \varepsilon^{2}\left\|u_{0}\left(x_{0}\right)\right\|_{1 / 2, \partial \omega} \\
& \leq c \varepsilon^{2}\|\varphi\|_{1 / 2, \Gamma_{R}} .
\end{aligned}
$$

- On $\partial \omega_{\varepsilon}$, let $\theta_{\varepsilon}(x)=\left(-u_{0}(x)+u_{0}\left(x_{0}\right)-\varepsilon R_{U}(x)\right) / \varepsilon$, we have $\operatorname{div} \theta_{\varepsilon}=0$ in $D_{0}$ and for small $\varepsilon$

$$
\begin{aligned}
\left\|\theta_{\varepsilon}(\varepsilon y)\right\|_{1 / 2, \partial \omega} & \leq c\left\|\theta_{\varepsilon}(\varepsilon y)\right\|_{1, \omega} \\
& \leq c\left\|\frac{u_{0}(\varepsilon y)-u_{0}\left(x_{0}\right.}{\varepsilon}+R_{U}(\varepsilon y)\right\|_{1, \omega} \\
& \leq c\left(\left\|u_{0}\right\|_{\mathcal{C}^{2}(B(0, R / 2))}+\left\|R_{U}\right\|_{\mathcal{C}^{1}(B(0, R / 2))}\right) \\
& \leq c\left(\|\varphi\|_{1 / 2, \Gamma_{R}}+\left\|S_{U}\right\|_{1 / 2, \Gamma_{R}}\right) \\
& \leq c\|\varphi\|_{1 / 2, \Gamma_{R}} .
\end{aligned}
$$

Then, using Lemmas 7.4 and 7.1 we get

$$
\begin{aligned}
\left|w_{\varepsilon}\right|_{1, C(R / 2, R)} & \leq c\left(\varepsilon^{2}\|\varphi\|_{1 / 2, \Gamma_{R}}+\varepsilon\left\|\theta_{\varepsilon}(\varepsilon y)\right\|_{1 / 2, \partial \omega}+\alpha\left\|L_{U}(x / \varepsilon)\right\|_{0, D_{\varepsilon}}\right) \\
& \leq c \varepsilon^{3 / 2}\|\varphi\|_{1 / 2, \Gamma_{R}} .
\end{aligned}
$$

Now, noting that $\operatorname{div}\left(w_{\varepsilon}(x)-L_{U}(x / \varepsilon)\right)=0$ in $C(R / 2, R)$ and $w_{\varepsilon}(x)=L_{U}(x / \varepsilon)$ on $\Gamma_{R}$.

Then, from the fact that

$$
\alpha\left(w_{\varepsilon}(x)-L_{U}(x / \varepsilon)\right)-\nu \Delta\left(w_{\varepsilon}(x)-L_{U}(x / \varepsilon)\right)+\nabla\left(s_{\varepsilon}(x)-1 / \varepsilon L_{P}(x / \varepsilon)\right)=\alpha L_{U}(x / \varepsilon) \text { in } C(R / 2, R),
$$

we deduce

$$
\begin{aligned}
\|\psi\|_{-1 / 2, \Gamma_{R}} & =\left\|\sigma\left(w_{\varepsilon}(x)-L_{U}(x / \varepsilon)\right) . n\right\|_{-1 / 2, \Gamma_{R}} \\
& \leq c\left(\left|w_{\varepsilon}(x)-L_{U}(x / \varepsilon)\right|_{1, C(R / 2 R)}+\left\|L_{U}(x / \varepsilon)\right\|_{0, C(R / 2 R)}\right) .
\end{aligned}
$$


Finally, due to (106), (81), (82) and Lemma 7.1, we obtain

$$
\|\psi\|_{-1 / 2, \Gamma_{R}} \leq c \varepsilon^{3 / 2}\|\varphi\|_{1 / 2, \Gamma_{R}} .
$$

Hence

$$
\left\|\left(T_{\varepsilon}-T_{0}-\varepsilon \delta T\right) \varphi\right\|_{1 / 2, \Gamma_{R}}=O\left(\varepsilon^{3 / 2}\right) .
$$

Proposition 7.2. Let

$$
\delta a(u, v)=\int_{\Gamma_{R}} \delta T u . v \mathrm{~d} \gamma(x) \quad \forall u, v \in \mathcal{V}_{R} .
$$

The asymptotic expansion of the linear form $a_{\varepsilon}$ is given by

$$
\left\|a_{\varepsilon}-a_{0}-\varepsilon \delta a\right\|_{\mathcal{L}_{2}\left(\mathcal{V}_{R}\right)}=O\left(\varepsilon^{3 / 2}\right) .
$$

\subsection{Variation of the linear form}

We search now to compute the variation of the linear form $l_{\varepsilon}$. For that purpose, we will use the same technique as in the preceding section. The unique difference comes from the boundary condition imposed on $\partial \omega$ to the solution of the exterior problem. Indeed, for the study of the bilinear form we have used $U^{0, \varphi}=u_{0}^{0, \varphi}\left(x_{0}\right)$ but for the study of the linear form we will use $U^{f, 0}=u_{0}^{f, 0}\left(x_{0}\right)$. As consequence, estimations involving $\|\varphi\|_{1 / 2, \Gamma_{R}}$ will be replaced by estimations involving $\|f\|_{2, D_{0}}$.

The variation of the linear form $l_{\varepsilon}$ reads

$$
l_{\varepsilon}(v)-l_{0}(v)=\int_{\Gamma_{R}}\left(f_{\varepsilon}-f_{0}\right) \cdot v \mathrm{~d} \gamma(x) .
$$

First, we denote by $\left(U^{f, 0}, P^{f, 0}\right)$ the solution to the exterior problem (83) corresponding to the boundary condition $\phi=u_{0}^{f, 0}\left(x_{0}\right)$ on $\partial \omega$, with $u_{0}^{f, 0}\left(x_{0}\right)$ is the solution to (13) or (14) if $\varepsilon=0$.

Using the same decomposition like in (38), we have

$$
U^{f, 0}=S_{U}^{f, 0}+L_{U}^{f, 0}, \quad P^{f, 0}=S_{P}^{f, 0}+L_{P}^{f, 0}
$$

with $S_{U}^{f, 0}(y)=G_{U}(y) A\left(u_{0}^{f, 0}\left(x_{0}\right)\right), S_{P}^{f, 0}(y)=G_{P}(y) A\left(u_{0}^{f, 0}\left(x_{0}\right)\right)$.

Now, let $\left(R_{U}^{f, 0}, R_{P}^{f, 0}\right)$ be the associated solution to (41) with $R_{U}^{f, 0}=S_{U}^{f, 0}$ on $\Gamma_{R}$.

Then the linear form $\delta f$ (independent of $\varepsilon$ ) is given by

$$
\delta f=\sigma\left(R_{U}^{f, 0}-S_{U}^{f, 0}\right) \cdot n .
$$

Proposition 7.3. Let $f \in H^{2}(\Omega)^{3}$. The asymptotic expansion of $f_{\varepsilon}$ is given by

$$
\left\|f_{\varepsilon}-f_{0}-\varepsilon \delta f\right\|_{-1 / 2, \Gamma_{R}}=O\left(\varepsilon^{3 / 2}\right) .
$$

Proof. We use the same proof as in Proposition 7.1 with

$$
\begin{aligned}
w_{\varepsilon} & =u_{\varepsilon}^{f, 0}-u_{0}^{f, 0}+U^{f, 0}(x / \varepsilon)-\varepsilon R_{U}^{f, 0}, \\
\theta_{\varepsilon}(x) & =\left(-u_{0}^{f, 0}(x)+u_{0}^{f, 0}\left(x_{0}\right)-\varepsilon R_{U}^{f, 0}(x)\right) / \varepsilon .
\end{aligned}
$$

In this case, elliptic regularity implies

$$
\left|u_{0}^{f, 0}\left(x_{0}\right)\right| \leq\left\|u_{0}^{f, 0}\right\|_{\mathcal{C}^{0}\left(\mathcal{D}_{0}\right)} \leq\left\|u_{0}^{f, 0}\right\|_{2, D_{0}} \leq c\|f\|_{0, D_{0}}
$$


and for small $\varepsilon$, we have

$$
\begin{aligned}
\left\|\theta_{\varepsilon}(\varepsilon y)\right\|_{1 / 2, \partial \omega} & \leq c\left\|\theta_{\varepsilon}(\varepsilon y)\right\|_{1, \omega} \\
& \leq c\left\|\frac{u_{0}^{f, 0}(\varepsilon y)-u_{0}^{f, 0}\left(x_{0}\right.}{\varepsilon}+R_{U}^{f, 0}(\varepsilon y)\right\|_{1, \omega} \\
& \leq c\left(\left\|u_{0}^{f, 0}\right\|_{\mathcal{C}^{2}(B(0, R / 2))}+\left\|R_{U}^{f, 0}\right\|_{\mathcal{C}^{1}(B(0, R / 2))}\right) \\
& \leq c\|f\|_{2, D_{0}} .
\end{aligned}
$$

Proposition 7.4. Let $\delta l(v)=\int_{\Gamma_{R}} \delta f . v \mathrm{~d} \gamma(x), \quad v \in \mathcal{V}_{R}$.

The asymptotic expansion of $l_{\varepsilon}$ is given by

$$
\left\|l_{\varepsilon}-l_{0}-\varepsilon \delta l\right\|_{-1 / 2, \Gamma_{R}}=O\left(\varepsilon^{3 / 2}\right) .
$$

\subsection{Proof of Theorem $\mathbf{5 . 1}$}

Thanks to the previous results given in Propositions 7.2 and 7.4, we deduce that the hypotheses (1) and (2) hold. Then, we are now ready to apply the tools of Section 2. So that, from Theorem 2.1 we have

$$
j(\varepsilon)=j(0)+\varepsilon\left[\delta a\left(u_{0}, v_{0}\right)-\delta l\left(v_{0}\right)+\delta J\left(u_{0}\right)\right]+o(\varepsilon) .
$$

Due to (31), (98) and (110) we derive

$$
U=U^{f, 0}+U^{0, \varphi}
$$

and

$$
\begin{aligned}
& S_{U}=S_{U}^{f, 0}+S_{U}^{0, \varphi}, \\
& R_{U}=R_{U}^{f, 0}+R_{U}^{0, \varphi} .
\end{aligned}
$$

Then, using (100), (111), Propositions 7.2 and 7.4, we obtain

$$
\begin{aligned}
\delta a\left(u_{0}, v_{0}\right)-\delta l\left(v_{0}\right) & =\int_{\Gamma_{R}} \sigma\left(R_{U}^{0, \varphi}-S_{U}^{0, \varphi}\right) \cdot n v_{0} \mathrm{~d} \gamma(x)+\int_{\Gamma_{R}} \sigma\left(R_{U}^{f, 0}-S_{U}^{f, 0}\right) \cdot n v_{0} \mathrm{~d} \gamma(x) \\
& =\int_{\Gamma_{R}}\left[\left(\nu \nabla R_{U}^{f, \varphi}-R_{P}^{f, \varphi} I\right)-\left(\nu \nabla S_{U}^{f, \varphi}-S_{P}^{f, \varphi} I\right)\right] \cdot n v_{0} \mathrm{~d} \gamma(x) \\
& =\int_{\Gamma_{R}} \sigma\left(R_{U}^{f, \varphi}-S_{U}^{f, \varphi}\right) \cdot n v_{0} \mathrm{~d} \gamma(x) .
\end{aligned}
$$

This ends the proof of the theorem.

\section{REFERENCES}

[1] M. Abdelwahed, M. Amara, F. El Dabaghi and M. Hassine, A numerical modelling of a two phase flow for water eutrophication problems. ECCOMAS 2000, European Congress on Computational Methods in Applied Sciences and Engineering, Barcelone, 11-14 September (2000).

[2] G. Allaire and R. Kohn, Optimal bounds on the effective behavior of a mixture of two well-order elastic materials. Quat. Appl. Math. 51 (1993) 643-674.

[3] M. Bendsoe, Optimal topology design of continuum structure: an introduction. Technical report, Department of mathematics, Technical University of Denmark, DK2800 Lyngby, Denmark, September (1996).

[4] F. Brezzi and M. Fortin, Mixed and hybrid finite element method. Springer Ser. Comput. Math. 15 (1991).

[5] G. Buttazzo and G. Dal Maso, Shape optimization for Dirichlet problems: Relaxed formulation and optimality conditions. Appl. Math. Optim. 23 (1991) 17-49.

[6] J. Céa, A. Gioan and J. Michel, Quelques résultats sur l'identification de domaines. CALCOLO (1973). 
[7] J. Céa, Conception optimale ou identification de forme, calcul rapide de la dérivée directionnelle de la fonction coût. ESAIM: M2AN 20 (1986) 371-402.

[8] J. Céa, S. Garreau, Ph. Guillaume and M. Masmoudi, The shape and Topological Optimizations Connection. Comput. Methods Appl. Mech. Engrg. 188 (2000) 713-726.

[9] M. Chipot and G. Dal Maso, Relaxed shape optimization: the case of nonnegative data for the Dirichlet problems. Adv. Math. Sci. Appl. 1 (1992) 47-81.

[10] P. Ciarlet, The Finite Element Method for Elliptic Problems. North-Holland (1978).

[11] R. Dautray et J. Lions, Analyse mathémathique et calcul numérique pour les sciences et les techniques. Masson, collection CEA (1987).

[12] J. Douglas and T.F. Russell, Numerical methods for convection dominated diffusion problems based on combining the method of characteristics with finite element methods or finite difference method. SIAM J. Numer. Anal. 19 (1982) 871-885.

[13] M. Fortin, R. Peyret et R. Temam, Résolution numérique des équations de Navier-Stokes pour un fluide incompressible. J. Mécanique 10 (1971).

[14] S. Garreau, Ph. Guillaume and M. Masmoudi, The topological sensitivity for linear isotropic elasticity. European Conferance on Computationnal Mechanics (1999) (ECCM99), report MIP 99.45.

[15] S. Garreau, Ph. Guillaume and M. Masmoudi, The topological asymptotic for pde systems: the elasticity case. SIAM J. Control Optim. 39 (2001) 1756-1778.

[16] P. Germain and P. Muller, Introduction à la mécanique des milieux continus. Masson (1994).

[17] V. Girault and P.A. Raviart, Finite element methods for Navier-Stokes equations, Theory and Algorithms. Springer-Verlag Berlin (1986).

[18] J. Giroire, Formulations variationnelles par équations intégrales de problèmes aux limites extérieurs. Thèse, École Polytechnique, Palaiseau (1976).

[19] R. Glowinski, Numerical methods for nonlinear variational problems. J. Optim. Theory Appl. 57 (1988) 407-422.

[20] R. Glowinski and O. Pironneau, Toward the computational of minimun drag profile in viscous laminar flow. Appl. Math. Model. 1 (1976) 58-66.

[21] P. Grisvard, Elliptic problems in non smooth domains. Pitman Publishing Inc., London (1985).

[22] Ph. Guillaume and M. Masmoudi, Computation of high order derivatives in optimal shape design. Numer. Math. 67 (1994) 231-250.

[23] Ph. Guillaume and K. Sid Idris, The topological asymptotic expansion for the Dirichlet Problem. SIAM J. Control. Optim. 41 (2002) 1052-1072.

[24] Ph. Guillaume and K. Sid Idris, Topological sensitivity and shape optimization for the Stokes equations. Rapport MIP (2001) $01-24$.

[25] M. Hassine, Contrôle des processus d'aération des lacs eutrophes. Thesis, Tunis II University, ENIT, Tunisia (2003).

[26] J. Jacobsen, N. Olhoff and E. Ronholt, Generalized shape optimization of three-dimensional structures using materials with optimum microstructures. Technical report, Institute of Mechanical Engineering, Aalborg University, DK-9920 Aalborg, Denmark (1996).

[27] J.L. Lions and E. Magenes, Problèmes aux limites non homogènes et applications. Dunod (1996).

[28] M. Masmoudi, Outils pour la conception optimale de formes. Thèse d'État, Université de Nice (1987).

[29] M. Masmoudi, The topological asymptotic, in Computational Methods for Control Applications, H. Kawarada and J. Periaux Eds., International Séries GAKUTO (2002).

[30] F. Murat and L. Tartar, Calcul des variations et homogénéisation, in Les méthodes de l'homogénéisation : Théorie et applications en physique. Eyrolles (1985) 319-369.

[31] O. Pironneau, Méthode des éléments finis pour les fluides. Masson, Paris (1988).

[32] O. Pironneau, Optimal Shape Design for Elliptic Systems. Springer, Berlin (1984).

[33] J. Simon, Domain variation for Stokes flow. X. Li and J. Yang Eds., Springer, Berlin, Lect. Notes Control Inform. Sci. 159 28-42 (1990)

[34] J. Simon, Domain variation for drag Stokes flows. A. Bermudez Eds., Springer, Berlin, Lect. Notes Control Inform. Sci. 114 (1987) 277-283.

[35] A. Schumacher, Topologieoptimierung von bauteilstrukturen unter verwendung von lopchpositionierungkrieterien. Thesis, Universitat-Gesamthochschule-Siegen (1995).

[36] M. Shœenauer, L. Kallel and F. Jouve, Mechanical inclusions identification by evolutionary computation. Rev. Eur. Élém. Finis 5 (1996) 619-648.

[37] J. Sokolowski and A. Zochowski, On the topological derivative in shape optimization. SIAM J. Control Optim. 37 (1999) 1251-1272 (electronic).

[38] R. Temam, Navier Stokes equations (1985). 